Check for updates

Cite this: J. Mater. Chem. B, 2020, 8,3852

\title{
Modified gaphene oxide (GO) particles in peptide hydrogels: a hybrid system enabling scheduled delivery of synergistic combinations of chemotherapeutics $\dagger$
}

\author{
John D. Schneible, (D) ${ }^{a}$ Kaihang Shi, ${ }^{a}$ Ashlyn T. Young, (D) ${ }^{b}$ Srivatsan Ramesh, ${ }^{a}$ \\ Nanfei He, (D) Clay E. Dowdey, ${ }^{\mathrm{a}}$ Jean Marie Dubnansky, ${ }^{a}$ Radina L. Lilova, ${ }^{a}$ \\ Wei Gao, ${ }^{c}$ Erik Santiso, ${ }^{a}$ Michael Daniele (D) *bd and Stefano Menegatti*a
}

\begin{abstract}
The scheduled delivery of synergistic drug combinations is increasingly recognized as highly effective against advanced solid tumors. Of particular interest are composite systems that release a sequence of drugs with defined kinetics and molar ratios to enhance therapeutic effect, while minimizing the dose to patients. In this work, we developed a homogeneous composite comprising modified graphene oxide (GO) nanoparticles embedded in a Max8 peptide hydrogel, which provides controlled kinetics and molar ratios of release of doxorubicin (DOX) and gemcitabine (GEM). First, modified GO nanoparticles (tGO) were designed to afford high DOX loading and sustained release $(18.9 \%$ over $72 \mathrm{~h}$ and $31.4 \%$ over 4 weeks). Molecular dynamics simulations were utilized to model the mechanism of DOX loading as a function of surface modification. In parallel, a Max8 hydrogel was developed to release GEM with faster kinetics and achieve a 10-fold molar ratio to DOX. The selected DOX/tGO nanoparticles were suspended in a GEM/Max 8 hydrogel matrix, and the resulting composite was tested against a triple negative breast cancer cell line, MDA-MB-231. Notably, the composite formulation afforded a combination index of $0.093 \pm 0.001$, indicating a much stronger synergism compared to the DOX-GEM combination co-administered in solution $(\mathrm{Cl}=0.396 \pm 0.034)$.
\end{abstract}

Received 8th January 2020, Accepted 2nd March 2020

DOI: $10.1039 / \mathrm{d} 0 \mathrm{tb} 00064 \mathrm{~g}$

rsc.li/materials-b

\section{Introduction}

Combination chemotherapy is currently the most widely utilized, clinically established route to fight cancer, owing to its distinct advantages over current single-drug chemotherapy. Single-drug therapies cause acquired drug resistance upon prolonged administration, ${ }^{1,2}$ which cause non-responsiveness to therapy and the need of increased dosage. ${ }^{3,4}$ This results in poorer quality of life during treatment and decreased life expectancy. ${ }^{4-6}$

\footnotetext{
${ }^{a}$ Department of Chemical and Biomolecular Engineering,

North Carolina State University, 911 Partners Way, Raleigh, North Carolina, USA. E-mail: smenega@ncsu.edu

${ }^{b}$ Joint Department of Biomedical Engineering, North Carolina State University - University of North Carolina Chapel Hill, North Carolina, USA ${ }^{c}$ Department of Textile Engineering, Chemistry, and Science, 1020 Main Campus Drive, Raleigh, North Carolina, USA

${ }^{d}$ Department of Electrical and Computer Engineering, North Carolina State University, 890 Oval Drive, Raleigh, North Carolina, USA. E-mail: mdaniel6@ncsu.edu $\dagger$ Electronic supplementary information (ESI) available: Short time scale DOX release from modified GO library, zeta potential - amine surface density relationship, DOX loading for tGO at various zeta potential and loading buffer composition, simulation snapshots of DOX loading, DOX release at $\mathrm{pH}$ 7.4, and confocal images of composite hydrogel. See DOI: 10.1039/d0tb00064g
}

Combination chemotherapy offers superior therapeutic outcomes by operating through the simultaneous disruption of multiple metabolic pathways in cancer cells; these provide three mechanistic outcomes: synergistic, additive, and potentiation. ${ }^{7}$ Among these mechanistic outcomes, synergism, whereby the efficacy of multiple chemotherapeutics administered together is greater than the sum of individual therapies administered independently, represents the most favorable therapeutic outcome. ${ }^{7}$ Historically, synergism has been attributed to the combination of the drugs utilized and their molar ratio in the chemotherapeutic cocktail. ${ }^{7,8}$ More recently, however, the administration schedule, that is the sequence and timing by which every drug is administered, has been shown to be critical - together with molar ratio - to achieve therapeutic synergism. ${ }^{9-11}$

Recent research on the optimization of molar ratio and schedule of chemotherapeutic regimens has demonstrated that a more favorable therapeutic outcome can be achieved relative to single-drug therapy. This has been well-documented in preclinical studies, both in vitro and in animal models, ${ }^{10,12-18}$ as well as in clinical settings. ${ }^{19-22}$ Current methods to administer scheduled combined chemotherapy rely on subsequent injections, which require prolonged patient hospitalization and extended 
observation by trained specialists, and result in higher medical costs and discomfort for patients. It is now anticipated that next-generation treatments will rely on engineered solutions, comprising multiple biocompatible materials that (i) are seamlessly integrated into stable systems and (ii) release the various therapeutic ingredients at tunable amounts and rates to provide the desired therapeutic sequence, ratio, and kinetics.

To this end, researchers have investigated a myriad of drug delivery systems (DDSs), ranging from nano-/micro-particles and liposomes, ${ }^{23,24}$ to polymer conjugates (PDC's) ${ }^{10,12,13,18,25,26}$ and hydrogels. ${ }^{14,27-29}$ Among these systems, nanoparticles and hydrogels possess distinct advantages, mainly loading capacity and tenability of release. ${ }^{30-36}$

A nanoscale carrier that has attracted considerable attention as a DDS is graphene oxide (GO). GO features a high specific surface area (up to $500 \mathrm{~m}^{2} \mathrm{~g}^{-1}$ ), ${ }^{37}$ which is ideal for loading large quantities of poorly bioavailable drugs, such as taxanes, anthracyclines, and camptothecan analogues. ${ }^{38-49}$ Additionally, the surface of GO features an abundance of carboxylic acids, epoxides, and hydroxyl groups that can be modified to introduce stimuli-responsive behavior, enable active targeting, and prolong circulation. ${ }^{31,50,51}$ Drug adsorption and release on native GO has also been studied in silico using model drugs doxorubicin (DOX), paclitaxel, 5-fluorouracil, and thioguanine. ${ }^{52-55}$ These simulations have proven very helpful in designing GO-based drug delivery systems by guiding the choice of type and degree of surface modification, and the loading conditions that maximize therapeutic efficacy.

Hydrogels with shear-thinning and thixotropic behavior are also of interest in drug delivery, as they can be easily introduced by direct injection into surgical cavities to form "depots" that provide sustained drug release. ${ }^{34,35}$ This bypasses the pharmacokinetic limitations inherent in many chemotherapeutic agents, as well as the bio-distribution limitations of intravenously administered systemic delivery systems. ${ }^{34,35,56-58}$ Owing to their flexible molecular architecture, hydrogels enable release kinetics that can easily be tuned by adjusting the polymer/water ratio in cross-linked gels. . $^{59,60 ~ " D e s i g n e r " ~ p e p t i d e s ~}$ have received strong interest to construct hydrogel-based DDSs. Designer peptides are inherently biocompatible, bio-degradable, and feature rapid gelation via hierarchal self-assembly. ${ }^{61}$ Gelation kinetics can be tuned effectively by varying the amino acid composition of the peptide and the aqueous environment $(\mathrm{pH}$, ionic strength, temperature). ${ }^{62-66}$ A designer peptide widely used is the Max8 sequence, constituted by VKVKVKVKV ${ }^{\mathrm{D} P P}$ TKVEVKVKV-NH ${ }_{2}$, wherein ${ }^{\mathrm{D}} \mathrm{P}$ is D-proline. The Max8 peptide is a stimuli-responsive peptide that is stable in aqueous solutions at low ionic strength and undergoes gelation in to physiological conditions by self-assembling into $3.2 \mathrm{~nm}$ diameter $\beta$-hairpin nanofibers. ${ }^{64}$ At low gel fractions $(0.5-2 \mathrm{w} / \mathrm{w})$ the Max8 hydrogel imposes little resistance to the transport of both small and large molecules, as demonstrated by Branco et al. with fluoresceindextran conjugates. ${ }^{63}$ In the context of scheduled delivery of multiple drugs, the high permeability of the hydrogel makes it an ideal depot for the release of the first drug in a sequential system; the limited control of the relative flux of multiple diffusing drugs, however, limits the applicability of hydrogels alone for scheduled combined chemotherapy. Rather, composite systems combining drug-loaded nanocarriers suspended in a drug-loaded peptide hydrogel hold great promise for precise delivery kinetics of multiple payloads. ${ }^{67-70}$

In this work, we demonstrate scheduled and synergistic release of combined chemotherapeutics through the development of a composite hydrogel system comprising DOX-loaded modified-GO nanoparticles suspended in a gemcitabine (GEM)loaded Max8 hydrogel (Fig. 1). The synergism of DOX and GEM has been extensively studied in terms of molar ratio and delivery kinetics, ${ }^{9,10,71-73}$ demonstrating that the combination is synergistic for molar ratios $(\mathrm{GEM}: \mathrm{DOX})>1$, and optimal synergism occurs at a ratio of $10: 1$, with GEM being administered prior to DOX. ${ }^{9,10}$ We initially investigated different types and levels of chemical modification to tune the surface charge and hydrophobicity of GO nanoparticles and evaluated the corresponding DOX loading and release at different values of solution $\mathrm{pH}$ and ionic strength. Notably, GO modification with tris(2-aminoethyl) amine (TREN) achieved high loading, ranging from 0.2-0.6 $\mathrm{mg}$ DOX per $\mathrm{mg}$ GO, and afforded an initially rapid release of $18.9 \%$ of the loaded DOX within $72 \mathrm{~h}$ followed by sustained release of $31.4 \%$ over the course of 4 weeks. To gain molecular-level insight into the phenomena governing the adsorption and release of DOX on TREN-GO, we developed a computational model that quantitatively describes the DOX:TREN-GO interaction at different modification degrees, in terms of (i) molarity of loading and release (i.e., water/GO partition coefficient of DOX), and (ii) reversible formation and adsorption/desorption of DOX aggregates. Selected DOX-TREN-GO nanoparticles were then suspended in a GEM/Max8 hydrogel, and the composite formulation was evaluated on a triple negative breast cancer cell line (MDAMB-231) demonstrating high therapeutic efficacy. Notably, the DOX-GEM-loaded composite system afforded a combination index of $0.093 \pm 0.001$, considerably lower than the free drug DOX-GEM combination $(\mathrm{CI}=0.396 \pm 0.034)$ at the same concentration and molar ratio (DOX:GEM $=1: 10$ ). This indicates that the differential release induced from the composite system provides the desired therapeutic increment above the molar-based synergism.

\section{Experimental}

\section{Materials}

Graphite powder (4827) was sourced from Asbury Graphite Mills (Lumberton, NC). Doxorubicin hydrochloride (DOX) and Gemcitabine hydrochloride (GEM) were obtained from LC Laboratories (Woburn, MA). The Max8 peptide (VKVKVKVK $\mathrm{V}^{\mathrm{D}}$ PPTKVEVKVKV-NH 2 ), ${ }^{63,74,75}$ where ${ }^{\mathrm{D}} \mathrm{P}$ represents D-Proline and $-\mathrm{NH}_{2}$ indicates an amidated C-terminus, was obtained from Genscript (Nanjing, China) at $>95 \%$ purity. 1,1-Carbonyldiimidazole (CDI), dimethylaminopyridine (DMAP), ethylenediamine (EDA), tris(2-aminoethyl) amine (TREN), acetic anhydride $\left(\mathrm{Ac}_{2} \mathrm{O}\right)$, benzylamine (BA), succinic anhydride (SA), anhydrous 

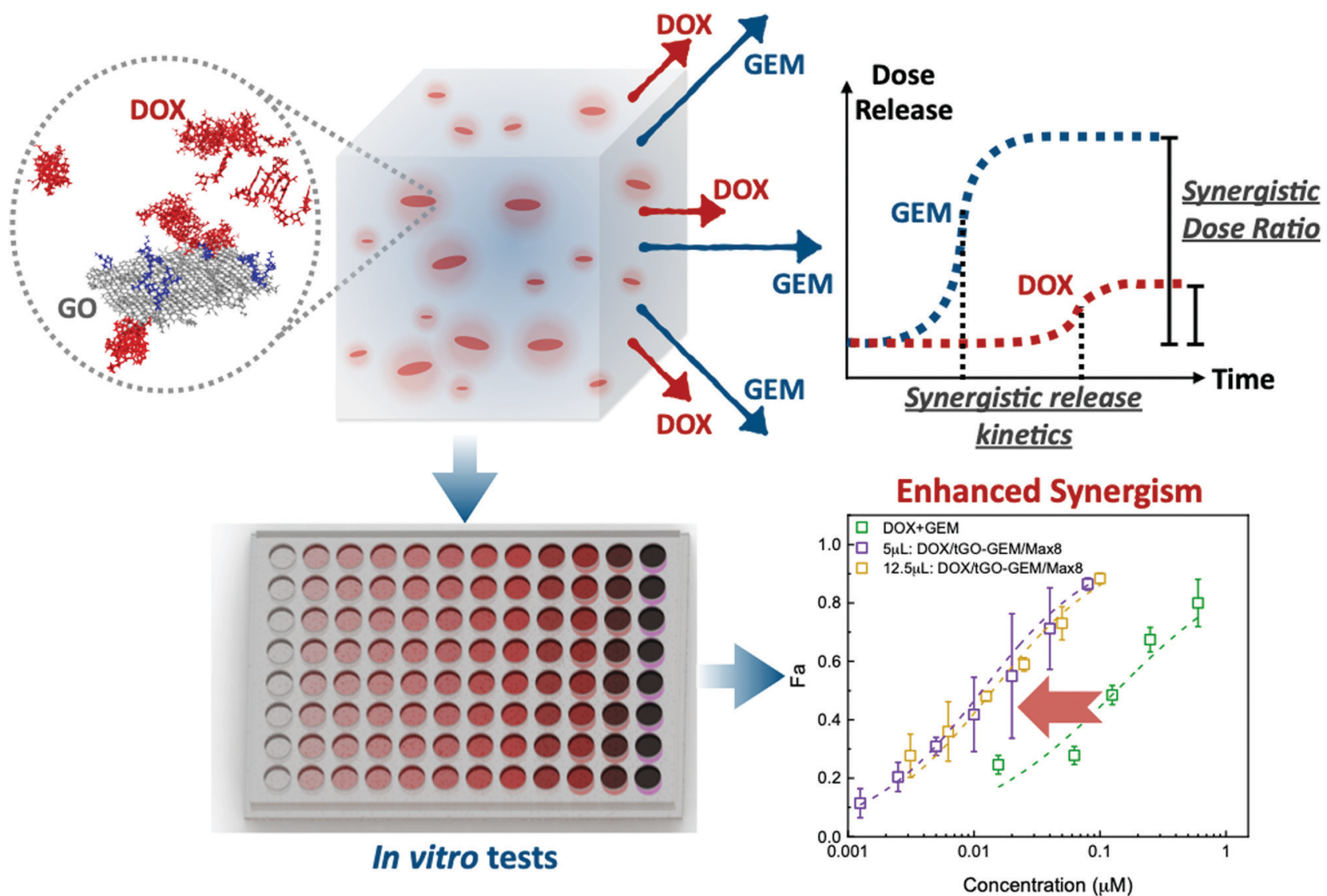

Fig. 1 Design and proposed mechanism of dual release of DOX and GEM from the hybrid system comprising modified GO nanoparticles suspended in a Max8 hydrogel. The combination of drug release at controlled relative molar ratio and kinetics enhances the inherent synergism of DOX and GEM beyond the simple effect of codelivery.

tetrahydrofuran (THF), phosphate buffered saline (PBS), 4-(2hydroxyethyl)-1-piperazineethanesulfonic acid (HEPES), potassium permanganate $\left(\mathrm{KMnO}_{4}\right)$, and hydrogen peroxide $\left(\mathrm{H}_{2} \mathrm{O}_{2}\right)$ were purchased from Sigma (St. Louis, MO). Triple negative breast cancer cells MDA-MB-231 were purchased from ATCC (Manassas, VA). Dulbecco's Modified Eagle Medium (DMEM). Fetal bovine serum (FBS) was obtained from Genesee Scientific (San Diego, CA). Penicillin Streptomycin (Pen Strep) was obtained from Gibco (Gaithersburg, MD). 3-(4,5-Dimethylthiazol-2-yl)-2,5-diphenyltetrazolium bromide (MTT), was purchased from Invitrogen (Carlsbad, CA). All other chemicals were of reagent grade or higher.

\section{Synthesis and characterization of modified graphene oxide (GO)}

Synthesis of GO particles. GO was synthesized from pretreated graphite powder (4827) following the modified Hummers' method. ${ }^{25,76}$ Briefly, graphite $(6 \mathrm{~g})$ was added into $\mathrm{H}_{2} \mathrm{SO}_{4}$ $(230 \mathrm{~mL})$, which was placed into a $1 \mathrm{~L}$ Erlenmeyer flask and cooled to $<10{ }^{\circ} \mathrm{C}$ using an ice bath. Then, $\mathrm{KMnO}_{4}(30 \mathrm{~g})$ was added slowly to the mixture and kept stirred continually. The mixture was then allowed to react at room temperature (RT) for 2 hours. Afterwards, distilled (DI) water $(460 \mathrm{~mL})$ and $30 \% \mathrm{H}_{2} \mathrm{O}_{2}$ $(50 \mathrm{~mL})$ were added slowly into the mixture, resulting in a brilliant yellow color. The mixture was allowed to settle for three days after which the clear supernatant was decanted. The remaining mixture was washed with $\mathrm{HCl}(10 \% \mathrm{v} / \mathrm{v})$ solution followed by DI water until the $\mathrm{pH}$ of the dispersion reached about 5 . The resulting GO dispersion was dried in air, and then dispersed at $5 \mathrm{mg} \mathrm{mL}^{-1}$ in anhydrous THF and sonicated for $30 \mathrm{~min}$. The GO was finally washed once with THF, acetone, and water, lyophilized, and stored for subsequent modifications. For washing steps, the GO suspension was centrifuged at $5000 \mathrm{rpm}$ for $5 \mathrm{~min}$; minor loss of GO particles was observed during washing steps $(<5 \%$ measured by weight of dry particles). The size of GO nanoparticles was determined by scanning electron microscopy (SEM) using a FEI Verios 460L (Philips) on a dilute aqueous solution $\left(<0.1 \mathrm{mg} \mathrm{mL}^{-1}\right)$ drop cast on aluminum foil.

Surface modification of GO particles. Lyophilized GO was initially dispersed at $5 \mathrm{mg} \mathrm{mL}{ }^{-1}$ in anhydrous THF via sonication for $30 \mathrm{~min}$. For surface modification with amines, CDI $(50 \mathrm{mg}$ ) was added and allowed to react for $1 \mathrm{~h}$ RT. The corresponding amine ( 0.5 equiv. of TREN, EDA, or BA) was then added to the activated GO suspension and allowed to react for $1 \mathrm{~h}$ at RT. To saturate unreacted groups, ethanolamine was added to the GO nanoparticles at a concentration in solution of $0.1 \mathrm{M}$ and allowed to react for $1 \mathrm{~h}$ at RT. The GO was then washed with THF, acetone, and water, and lyophilized. For surface 
acylation with anhydrides, the corresponding anhydride ( 0.5 equiv. of $\mathrm{Ac}_{2} \mathrm{O}$ or $\mathrm{SA}$ ) and catalytic DMAP were added to the GO suspension and allowed to react for $24 \mathrm{~h}$ at RT. To saturate unreacted groups, ethanolamine was added to the GO nanoparticles at a concentration in solution of $0.1 \mathrm{M}$ and allowed to react for $1 \mathrm{~h}$ at RT. The GO particles were then washed with THF, acetone, and water, and lyophilized. The resulting samples were analyzed using a Zetasizer Nano (Malvern, UK) to determine the zeta potential ( $\zeta$ potential) of the modified GO particles.

DOX loading and release screening studies. All GO samples were incubated in $0.5 \mathrm{~mL}$ of aqueous DOX solution $\left(1 \mathrm{mg} \mathrm{mL}^{-1}\right)$ for $48 \mathrm{~h}$ at RT. Following incubation, the samples were centrifuged and the concentration of DOX in the supernatant was measured by UV-vis spectroscopy at $480 \mathrm{~nm}$ using a Synergy microplate reader (Biotek, Winooski, VT). The DOX-loaded GO particles were rinsed with water (to remove loosely bound DOX) and lyophilized. Release experiments were conducted utilizing a mass of particles corresponding to an equivalent mass of DOX (0.182 mg). The particles were suspended in $1 \mathrm{~mL}$ of PBS, $\mathrm{pH}$ 7.4 at $37^{\circ} \mathrm{C}$. At assigned time points $(12,24,48,72,96,144,192$, 288, 336, 384, 432, $504 \mathrm{~h}), 200 \mu \mathrm{L}$ of supernatant was collected and replenished with fresh PBS. The samples were then analyzed by UV-vis spectroscopy at $480 \mathrm{~nm}$ to determine the amount of DOX released.

\section{Synthesis and characterization of TREN-GO (tGO) library}

Synthesis of (tGO) particle library. Lyophilized GO was initially dispersed in anhydrous THF at $5 \mathrm{mg} \mathrm{mL}^{-1}$ by sonication for $30 \mathrm{~min}$. Aliquots of $1 \mathrm{~mL}$ of GO suspension were combined with $50 \mathrm{mg}$ of CDI and allowed to react for $1 \mathrm{~h} \mathrm{RT}$. A variable volume of TREN (0.25-2 equiv., relative to CDI) was then added and allowed to react for $1 \mathrm{~h}$ at RT to generate TREN-modified GO particles (tGO) with different surface density. To saturate unreacted groups, ethanolamine was added to the GO nanoparticles at a concentration in solution of $0.1 \mathrm{M}$ and allowed to react for $1 \mathrm{~h}$ at RT. The tGO particles were then washed with THF, acetone, and water, and lyophilized. The resulting samples were analyzed using a Malvern Zetasizer Nano to determine the $\zeta$ potential of the modified tGO particles, and a modified Kaiser's colorimetric test the surface density of primary amines. ${ }^{77}$ For Kaiser's test, $100 \mu \mathrm{L}$ of tGO particles suspension $\left(0.1 \mathrm{mg} \mathrm{mL}^{-1}\right.$ in $\left.\mathrm{H}_{2} \mathrm{O}\right)$ were combined with $30 \mu \mathrm{L}$ of potassium cyanide in water/pyridine and ninhydrin, and $30 \mu \mathrm{L}$ of $6 \%$ ninhydrin in ethanol, and incubated at $100{ }^{\circ} \mathrm{C}$ for $5 \mathrm{~min}$. The solutions were diluted 200 -fold and analyzed by UV-vis spectroscopy at $570 \mathrm{~nm}$. Aqueous ethanolamine $\left(0.00125-0.025 \mathrm{mmol} \mathrm{mL}^{-1}\right)$ was used to generate the calibration curve.

Additionally, both GO and tGO particles were analyzed by Fourier Transform Infrared (FTIR) Spectroscopy using a Nicolet 6700 FTIR Spectrophotometer (ThermoFisher Scientific, Waltham, MA). First, the particles were finely ground in potassium bromide (dried in a vacuum oven for $3 \mathrm{~h}$ at $60{ }^{\circ} \mathrm{C}$ ) and placed in the optical bench, which was purged continuously using dry air. The samples were then analyzed using the following data acquisition parameters: accumulation of 512 interferograms with a resolution of $4 \mathrm{~cm}^{-1}$, wavenumber range of $4000-400 \mathrm{~cm}^{-1}$.
The resulting spectra were analyzed using the OMNIC Spectra Software (Thermo Scientific).

DOX loading and release from tGO particle library. DOX loading studies were performed on native GO and tGO in different aqueous environments, namely low ionic strength (0.1 $\mathrm{mM}$ at $\mathrm{pH} 4,1 \mu \mathrm{M}$ at $\mathrm{pH} 6$, and $0.1 \mathrm{nM}$ at $\mathrm{pH}$ 9; these values of concentration and $\mathrm{pH}$ were achieved by titration with aqueous hydrochloric acid or sodium hydroxide), and PBS at $\mathrm{pH}$ 7.4. Loading solutions were prepared by dissolving DOX at $1 \mathrm{mg} \mathrm{mL}{ }^{-1}$ in the different aqueous solvents. The solutions $(0.5 \mathrm{~mL})$ were incubated with $\mathrm{GO}$ and the various tGO samples (0.5 $\mathrm{mg}$ ) for $48 \mathrm{~h}$ at RT. After loading, the samples were centrifuged and the supernatants were analyzed by UV-vis spectroscopy at $480 \mathrm{~nm}$, to quantify the amount of residual DOX in solution. The DOX-loaded particles were rinsed with water, and lyophilized. Release tests were conducted by incubating a mass of particles corresponding to an equivalent mass of DOX $(0.1 \mathrm{mg})$ in $1 \mathrm{~mL}$ of $10 \mathrm{mM}$ PBS, at either pH 7.4 or pH 5 at $37^{\circ} \mathrm{C}$. At set time points, (72, 170, 263, 378, 500, $\left.650 \mathrm{~h}\right)$, $200 \mu \mathrm{L}$ of supernatants from the various samples were collected and replenished with fresh buffer. The collected samples were analyzed by UV-vis spectroscopy at $480 \mathrm{~nm}$ to determine the amount of DOX released.

Molecular dynamics (MD) simulations of tGO. To study the interactions between DOX molecules and the surface of native and TREN-modified GO at the molecular level, we adopted a simplified version of the constant-pH molecular dynamics (CpHMD) simulation technique by Baptista et al. ${ }^{78}$ In place of coupling with the stochastic protonation/deprotonation algorithm during the simulation, our version assigns a constant protonation or deprotonation to the titratable amine group of the daunosamine moiety of the DOX molecules throughout the entire simulation. This method has already been implemented in several studies. ${ }^{53,79}$ The number of protonated/deprotonated sites in GO sheet and DOX molecules was approximated using the Henderson-Hasselbalch equation. ${ }^{80,81}$ Assuming that the $\mathrm{p} K_{\mathrm{a}}$ values of isolated carboxyl groups $(\mathrm{COOH})$ and hydroxyl groups $(\mathrm{OH})$ on $\mathrm{GO}$ sheet are 6.6 and $9.8,{ }^{82}$ respectively, the number of deprotonated sites was calculated by:

$$
N_{\text {depro }}=\frac{10^{\mathrm{pH}-\mathrm{p} K_{\mathrm{a}}}}{1+10^{\mathrm{pH}-\mathrm{p} K_{\mathrm{a}}}} N_{\mathrm{t}}
$$

where $N_{\text {depro }}$ and $N_{\mathrm{t}}$ are the number of deprotonated sites and the total number of titratable carboxyl and hydroxyl groups, respectively. For DOX molecules, by assuming that the $\mathrm{p} K_{\mathrm{a}}$ value of the amino group $\left(-\mathrm{NH}_{2}\right)$ is $8.4,{ }^{83}$ the number of protonated DOX molecules was calculated by:

$$
N_{\text {pro }}=\frac{1}{1+10^{\mathrm{pH}-\mathrm{p} K_{\mathrm{a}}}} N_{\mathrm{t}}
$$

where $N_{\text {pro }}$ is the number of protonated DOX molecules. The adopted GO model has a chemical formula of $\mathrm{C}_{10} \mathrm{O}_{1}(\mathrm{OH})_{1}$ $(\mathrm{COOH})_{0.5}$ representing the standard oxidation process. ${ }^{84,85}$ Epoxy and hydroxyl groups are randomly distributed on the GO surface and the carboxyl groups are attached to the edge of the GO sheet. The general amber force field (GAFF) ${ }^{86}$ 
implemented in the Amber18 simulation package was used in all simulations. Partial charges of DOX and functional groups on the GO surface (i.e., epoxy group, hydroxyl group, and carboxyl group) were obtained using the standard AMBER charge fitting procedure. First, the geometry optimization of the molecule was performed at the DFT/B3LYP/6-31G* level, and then at MP2/6-31G(d) level with tight convergence criteria using Gaussian 09; the partial charges were then derived by fitting to the molecular electrostatic potential (MEP) at the HF/ 6-31G* level using the restrained electrostatic potential (RESP) method $^{87}$ through R.E.D. server. ${ }^{88,89}$ For the GO sheet, all $\mathrm{sp}^{2}$ carbon atoms were treated as uncharged, and atomic charges were only assigned to the atoms in a functional group and carbon atoms directly bonded to that functional group. The total charges of the molecule/fragment were set based on their protonated state in the solution. We ensured that the fitted RESP charges for the functional groups on the GO sheet agree with the AM1-BCC charges ${ }^{90,91}$ calculated by the AmberTool and with the scaled electrostatic potential (SESP) charges, ${ }^{92}$ which are linearly scaled to include the polarization effect in the aqueous solution; this agreement provided confidence in the current RESP charges, confirming the choice of $6-31 G^{*}$ basis set as sufficient to implicitly represent the polarization effect in the aqueous solution. ${ }^{87}$ We further confirmed that partial charges of protonated DOX molecules agreed with the reported data, ${ }^{93}$ and that the DOX structure obtained from quantum mechanics (QM) geometry optimization agrees with that from molecular mechanics (MM) energy minimization in implicit solvent by using the GAFF. We assigned all atoms types using Antechamber, ${ }^{94}$ and verified that the atom types assigned matches the true chemical environment of that atom. For simplicity, we manually changed all $\mathrm{sp}^{2}$ carbon atoms in the GO sheet to the "ca" type. The partial charges of the major functional groups on GO surface are summarized in Table 1.

To model the TREN-modified GO (tGO) surface, we tethered TREN moieties on the GO through carbamate bonds. While we recognized that TREN groups can be added to the surface of the particles through other functional groups on the native GO surface (e.g., hydroxyl groups and epoxy groups), we showed that our proposed scheme of TREN modification of the surface is sufficient to investigate the tGO-DOX interaction during both adsorption and release. The TREN density on the model tGO surface (molecules per $\mathrm{nm}^{2}$ ) was calculated from the experimental values measured by Kaiser's test, assuming $500 \mathrm{~m}^{2} \mathrm{~g}^{-1}$ as specific surface area for the GO particles. ${ }^{37}$ As the surface area of our GO model is $50 \mathrm{~nm}^{2}$, the number of TREN groups on the model surface ranges between 18 and 24 . The $\mathrm{p} K_{\mathrm{a}}$ values of the two primary amines and the tertiary amine on TREN are 10.6 and 10.9, respectively. Under weak neutral conditions $(\mathrm{pH}=6)$, all amine groups in the TREN are protonated and the TREN molecule carries a +3 charge. The initial configuration of the system was created using the PACKMOL package ${ }^{95}$ and the simulation box was filled using the TIP3P water model. ${ }^{96}$ Sixty-four DOX molecules were initially randomly distributed at a distance from the GO surface ranging between 0 and $45 \AA$ A. Following initial geometry optimization, the system was slowly heated from $0 \mathrm{~K}$ to room temperature $(298 \mathrm{~K})$ in an NVT ensemble for 200 ps with 2 fs time steps, while restraining the solid surface and the DOX molecules in their initial position by a harmonic spring force. The DOX molecules were then released, and an NPT simulation was performed to equilibrate the density of the system at 1 bar, $298 \mathrm{~K}$ for $2 \mathrm{~ns}$. Throughout the simulation, the pressure was maintained constant using the Berendsen barostat with pressure relaxation time of 2 ps, while the temperature was controlled using the Langevin thermostat with collision frequency $\gamma=1 \mathrm{ps}^{-1}$; the Langevin thermostat has been shown to be more efficient in system equilibration than the Berendsen temperature coupling scheme, but the Berendsen thermostat is more stable in reproducing the correct dynamics of the system. ${ }^{97}$ Starting from the last equilibrated frame, a production NPT MD was performed wherein all TREN groups on the surface were allow to relax while the base GO sheet was restrained using a weak

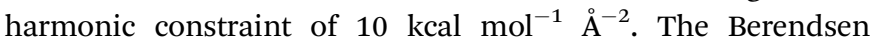
thermostat was used during the simulation with the temperature

Table 1 Partial charges for major functional groups

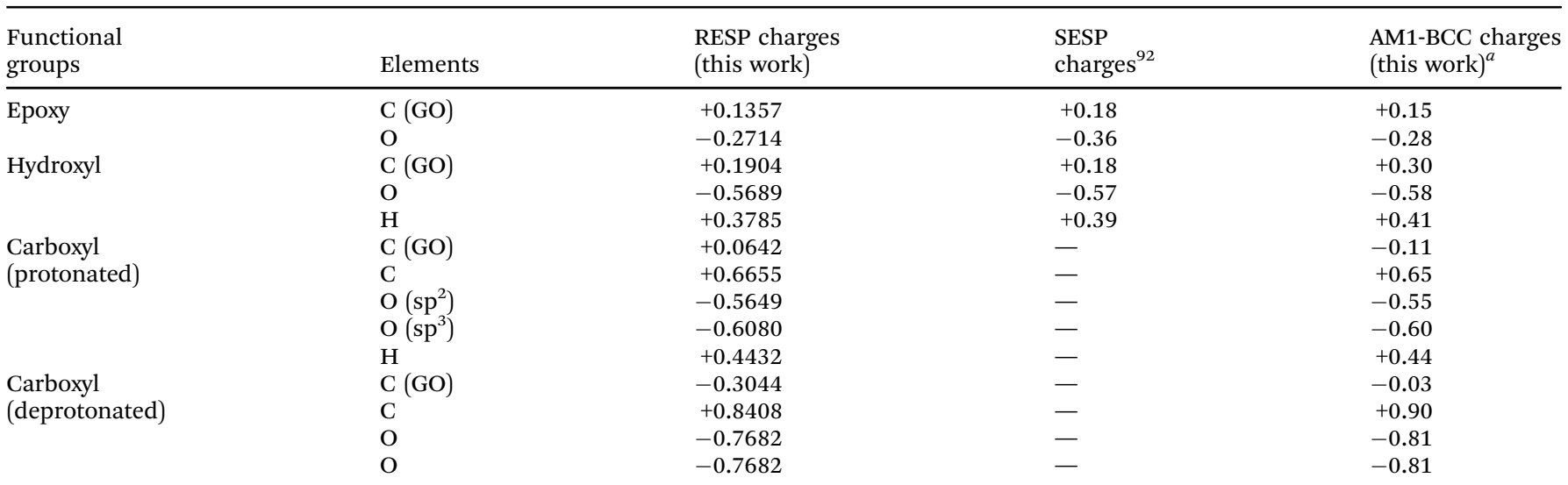

${ }^{a}$ AM1-BCC charges were determined for the whole molecule, so the total charge of the functional group fragment is not necessarily equal to 0 (neutral state) or -1 (deprotonated state). 
coupling time of $10 \mathrm{ps}$. All simulations were run over $70 \mathrm{~ns}$, at which time the number of DOX molecules adsorbed on either the native GO or tGO surface reaches a plateau. At least two independent MD simulations were run for the native GO and three tGO $\left(0.359,0.435\right.$, and 0.491 TREN molecules per $\mathrm{nm}^{2}$ corresponding to $\zeta$ potential of $-8.9,10.1$, and $20.7 \mathrm{mV}$, respectively) models to calculate the average number of DOX molecules adsorbed on different GO surfaces for subsequent comparison with experimental data.

\section{Synthesis and characterization of tGO-Max8 composite}

Preparation Max8 hydrogel and tGO-Max8 composite. All hydrogels were prepared at $2 \mathrm{w} / \mathrm{w}$ following published protocols. $^{75}$ Briefly, a peptide solution at $40 \mathrm{mg} \mathrm{mL}^{-1}$ in MilliQ water was combined with an equal volume of $100 \mathrm{mM}$ HEPES added with $300 \mathrm{mM} \mathrm{NaCl}, \mathrm{pH}$ 7.4. The solution was briefly vortexed and centrifuged, and allowed to rest until gelation (inversion test). This protocol was also adapted for release of GEM by initially dissolving the peptide in an aqueous solution of GEM at desired concentration. The tGO particles, tGO-DOX particles, Max8 peptide hydrogel, tGO particles suspended in Max8 peptide hydrogel (tGO-Max8), and tGO-DOX particles suspended in Max8 peptide hydrogel (DOX/tGOMax8) were imaged by fluorescent confocal microscopy using a Zeiss LSM 710 microscope (Carl Zeiss AG, Oberkochen, Germany) at $\lambda_{\mathrm{ex}}=590 \mathrm{~nm}$ and $\lambda_{\mathrm{em}}=618 \mathrm{~nm}$.

Dual drug release from tGO-Max8 composite. Selected DOX/ tGO particles comprising tGO $(\zeta$ potential $=-19 \mathrm{mV})$ loaded with DOX in $10 \mathrm{mM}$ PBS (pH 7.4) was utilized for all release studies under 3 conditions: free tGO-DOX particles, DOX/tGO particles embedded in a Max8 hydrogel, and DOX/tGO particles embedded in a Max8 hydrogel loaded with GEM. All release experiments were performed utilizing a volume of DOX-loaded particles corresponding to an equivalent mass of DOX (0.1 mg), and GEM (0.0605 mg, when GEM was utilized). These values of payload were selected to obtain the projected 10:1 GEM:DOX molar ratio in solution upon release, based on the data of DOX release obtained. Samples including gels were prepared using a total gel volume of $200 \mu \mathrm{L}$. All release tests were performed by contacting every system with $1 \mathrm{~mL}$ of $10 \mathrm{mM}$ PBS at pH 5 at $37^{\circ} \mathrm{C}$. At set time points $(8,16,32,48$, and $72 \mathrm{~h}), 200 \mu \mathrm{L}$ of supernatant was collected and replenished with fresh buffer. The collected samples were analyzed by liquid chromatography using a reverse-phase Aeris $3.6 \mu \mathrm{m} \mathrm{C18} \mathrm{column}(50 \times 4.6 \mathrm{~mm})$ installed on a Waters 2690 HPLC system (Waters, Milford, MA). The chromatographic method utilized a $5-100 \%$ gradient of acetonitrile $(0.1 \% \mathrm{v} / \mathrm{v}$ formic acid) in water $(0.1 \% \mathrm{v} / \mathrm{v}$ formic acid) over 10 minutes, while monitoring the effluent at $290 \mathrm{~nm}$ and $480 \mathrm{~nm}$ to monitor GEM and DOX, respectively. The concentrations of GEM and DOX were determined by peak-area integration of the resulting $290 \mathrm{~nm}$ and $480 \mathrm{~nm}$ chromatograms relative to the respective standard curves.

\section{In vitro characterization}

Cell culture. Tumorigenic cells, MDA-MB-231, were cultured in DMEM supplemented with $10 \%$ FBS and 1\% Pen-Strep in a humidified incubator at $37{ }^{\circ} \mathrm{C}$ and $5 \% \mathrm{CO}_{2}$.
Cell viability. Cells $\left(5 \times 10^{3}\right.$ cells per well $)$ were seeded in a 96-well plate and allowed to adhere overnight. Stock solutions of DOX in pure DMSO and GEM in sterile MilliQ water were diluted in cell culture media (DMEM supplemented with 10\% v/v FBS and $1 \% \mathrm{v} / \mathrm{v}$ Pen-Strep) such that the maximum concentration of DMSO or water did not exceed $0.5 \% \mathrm{v} / \mathrm{v}$. For the systems containing DOX/tGO particles, the concentration of particles used was such that the concentration of DOX released after $72 \mathrm{~h}$ matched the concentration of DOX utilized in cell viability studies conducted with free drug in solution. For systems containing Max8 hydrogel, the volumes of hydrogel utilized $(5,12.5$, and $25 \mu \mathrm{L}$ ) were prepared aseptically. After $72 \mathrm{~h}$ of treatment, the media was aspirated, and the cells were incubated in a solution of $0.5 \mathrm{mg} \mathrm{mL} \mathrm{m}^{-1} \mathrm{MTT}$ in cell culture media for $4 \mathrm{~h}$ at $37{ }^{\circ} \mathrm{C}$. The MTT solution was aspirated and DMSO was added to the wells and allowed to shake for 30 minutes to dissolve formazan crystals. Plate absorbance was measured at $540 \mathrm{~nm}$ using a Biotek Synergy microplate reader.

\section{Statistical analysis}

Statistical significance was determined by evaluation of the Student's $t$-test using Microsoft Excel. Statistical significance for samples was evaluated against the control test condition, and $p<0.05$ was considered significant. For analysis ${ }^{*},{ }^{*}$, and *** represent $p<0.05,0.01,0.001$, respectively.

\section{Results and discussion}

\section{Synthesis and characterization of modified graphene oxide} (GO)

DOX loading and release screening studies. An ensemble of GO particles was initially constructed by surface modification with different functional moieties to elucidate the effect of surface hydrophobicity (benzyl- vs. acetyl-groups) and electrostatic charge (carboxyl $v s$. amine groups) on the adsorption and release of DOX. Loading of DOX was proceeded over $48 \mathrm{~h}$ to reach adsorption equilibrium. The resulting values of drug loading (mg DOX per mg of modified GO particles) are summarized in Fig. 2A. DOX adsorption on carbon-rich surfaces (e.g., carbon nanotubes,) can reach high values owing to the dense $\pi-\pi$ interactions. ${ }^{98}$ Benzyl-GO $(\zeta$ potential $=-27.5 \mathrm{mV})$ particles provided the highest loading at $0.501 \pm 0.005 \mathrm{mg}$ of DOX per mg GO; the display of benzyl moieties on the GO surface through distal carboxylic acids may in fact provide additional binding sites for $\pi-\pi$ interactions with DOX to occur. DOX loading on native GO ( $\zeta$ potential $=-36.5 \mathrm{mV})$ was slightly lower, reaching $0.488 \pm 0.005 \mathrm{mg} \mathrm{mg}^{-1}$ GO. Other surface modifications afforded lower DOX loading; acetyl- $(\zeta$ potential $=$ $-26.5 \mathrm{mV}$ ) and succinyl- $(\zeta$ potential $=-27.3 \mathrm{mV})$ modified GO showed almost identical DOX binding capacity, $0.205 \pm$ $0.001 \mathrm{mg} \mathrm{mg}^{-1}$ GO and $0.204 \pm 0.031 \mathrm{mg} \mathrm{mg}^{-1}$ GO, respectively. Surface modification of GO particles with amines EDA and TREN increased the loading slightly to $0.255 \pm 0.054 \mathrm{mg} \mathrm{mg}^{-1} \mathrm{GO}$ and $0.307 \pm 0.040 \mathrm{mg} \mathrm{mg}^{-1} \mathrm{GO}$, respectively. Such increase in DOX loading seems counterintuitive, given that DOX is a positively 
(A)

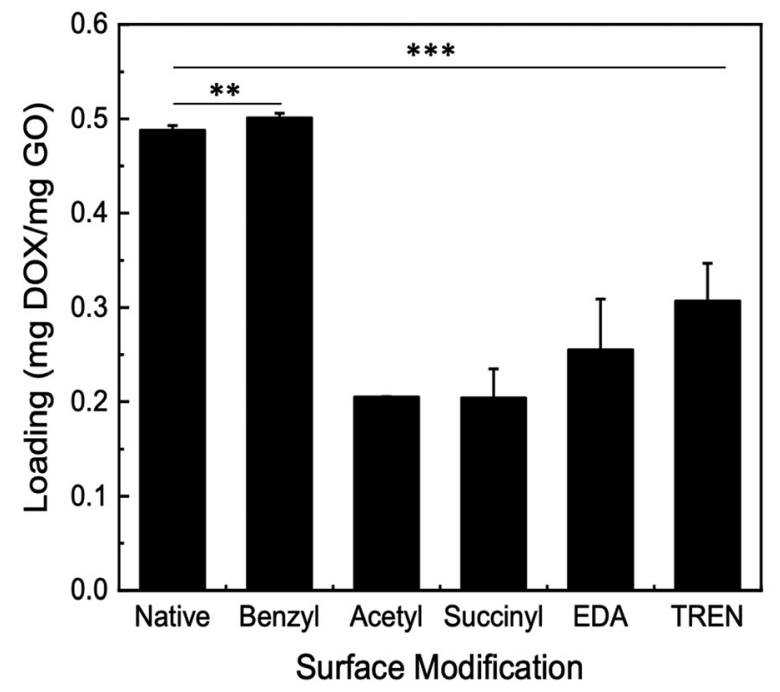

(B)

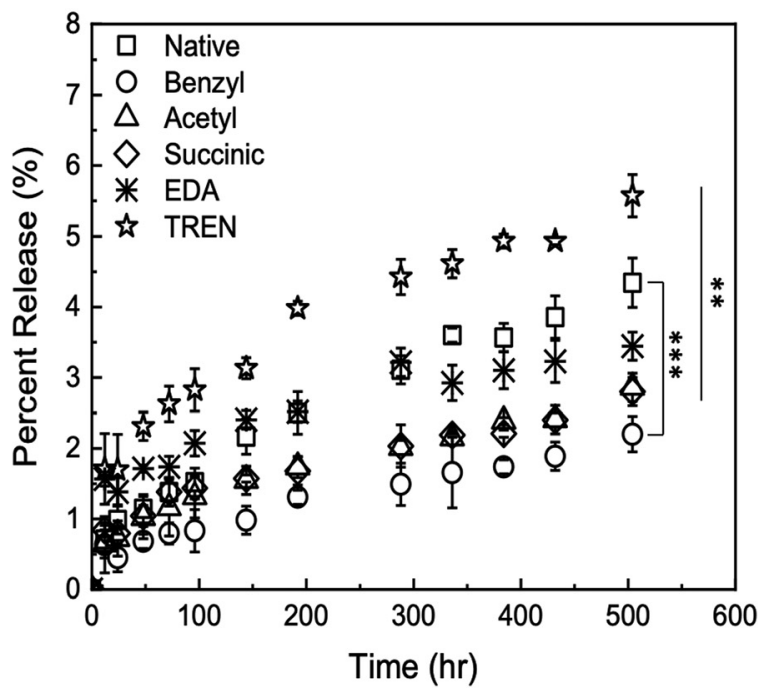

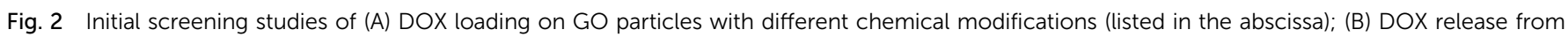

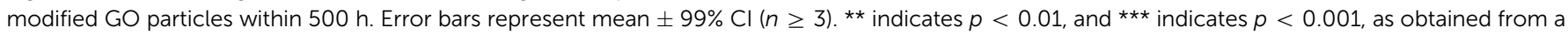
Student's t-test.

charged molecule and the surface charge of GO increases from $-26.5 \mathrm{mV}$ (acetyl-GO) to $\zeta$ potential $=-18.4 \mathrm{mV}(\mathrm{EDA})$ and $\zeta$ potential $=-14.3 \mathrm{mV}(\mathrm{TREN})$. This suggests that the interaction between DOX and modified GO does not depend solely on electrostatic interactions, but implicates other phenomena, such as steric hindrance upon adsorption, hydrogen bonding, and hydrophobic interaction, which are evaluated in detail later.

The release of DOX from the modified GO particles was performed in PBS at $\mathrm{pH} 7.4$ over a total collection time of $500 \mathrm{~h}$. The values of percent release (DOX released $v s$. loaded) are presented in Fig. 2B; the values of percent release obtained through the initial $100 \mathrm{~h}$ are presented in Fig. S2 (ESI $\dagger$ ). The GO particles modified with TREN and EDA returned the highest values of percent release, at both $72 \mathrm{~h}$ and $500 \mathrm{~h}$. The electrostatic repulsion between DOX and TREN/EDA moieties, both of which are positively charged at $\mathrm{pH}$ 7.4, combined with a high concentration gradient between the GO surface and the solution phase, likely play a predominant role in release. GO particles modified with TREN ( 2 primary and 1 tertiary amine), in fact, released $2.63 \pm 0.3 \%$ after $72 \mathrm{~h}$ and $5.57 \pm 0.3 \%$ after $504 \mathrm{~h}$, whereas GO modified with EDA (1 primary amine) released $1.74 \pm 0.2 \%$ after $72 \mathrm{~h}$ and $3.44 \pm 0.2 \%$ after $504 \mathrm{~h}$. Comparatively, at the $72 \mathrm{~h}$ and $504 \mathrm{~h}$ time points, native GO released $1.38 \pm 0.2 \%$ and $4.34 \pm 0.4 \%$, acetyl-GO released $1.16 \pm 0.4 \%$ and $2.86 \pm 0.2 \%$, succinyl-GO released $1.38 \pm$ $0.2 \%$ and $2.80 \pm 0.2 \%$, and benzyl-GO released $0.8 \pm 0.1 \%$ and $2.20 \pm 0.3 \%$. Notably, the loading of DOX on TREN-GO was only slightly lower compared to compared to that of native GO. These results indicate that modification of GO particles with cationic moieties is essential towards ensuring both a favorable loading and high release of DOX. Accordingly, we selected TREN as the surface modification moiety in all subsequent studies.
TREN-modified GO (tGO) characterization. Based on the initial screening process, we selected TREN-GO (tGO) as model particles in all subsequent studies of DOX loading and release. Prior to DOX adsorption and release studies, we investigated the correlation between the degree of TREN modification and the resulting electrostatic charge ( $\zeta$ potential potential) at the surface. To this end, we prepared an ensemble of tGO particles, and measured the TREN surface density using a modified Kaiser's amine quantification test and the corresponding values of $\zeta$ potential. The values of TREN density and $\zeta$ potential vs. molar ratio of TREN utilized in the modification reaction are presented in Fig. S3 (ESI $\dagger$ ). Notably, the ensemble of tGO particles encompassed a wide range of $\zeta$ potential, from $-36.5 \mathrm{mV}$ to $19.9 \mathrm{mV}$. The surface chemistry of native GO and one tGO sample ( $\zeta$ potential $=-18.8 \mathrm{mV}$ ) was also investigated by Fourier Transform InfraRed (FT-IR) spectroscopy (Fig. 3). The FT-IR spectrum of native GO exhibits the characteristic peaks of $\mathrm{O}-\mathrm{H}$ stretching at $3450 \mathrm{~cm}^{-1}, \mathrm{C}=\mathrm{O}$ stretching at $1736 \mathrm{~cm}^{-1}$, aromatic $\mathrm{C}=\mathrm{C}$ stretching at $1625 \mathrm{~cm}^{-1}$, alkoxy $\mathrm{C}-\mathrm{O}$ stretching at $1064 \mathrm{~cm}^{-1}$, and epoxy $\mathrm{C}-\mathrm{O}$ stretching at around $854 \mathrm{~cm}^{-1} \cdot{ }^{99}$ In tGO, the appearance of overlapping peaks of $\mathrm{C}=\mathrm{O}$ stretching and $\mathrm{N}-\mathrm{H}$ bending of the amide functional group at around $1640 \mathrm{~cm}^{-1}$, and the appearance of a C-N stretching peak at $1460 \mathrm{~cm}^{-1}$, 99 confirms the conjugation of TREN via formation of amide bonds. Furthermore, the presence of a carboxyl $\mathrm{C}=\mathrm{O}$ stretch peak at $1730 \mathrm{~cm}^{-1}$ in native GO and the absence of this peak in tGO indicates that all distal carboxylic acids are implicated in the formation of amide bonds with TREN. At the same time, the high values of TREN surface density measured on tGO particles and the exiguity of distal carboxylic acids typical of native GO also suggests that TREN conjugation also occurs through the formation of carbamate bonds, which result from the activation of the hydroxyl groups on GO with CDI; however, because carbamate and amide 


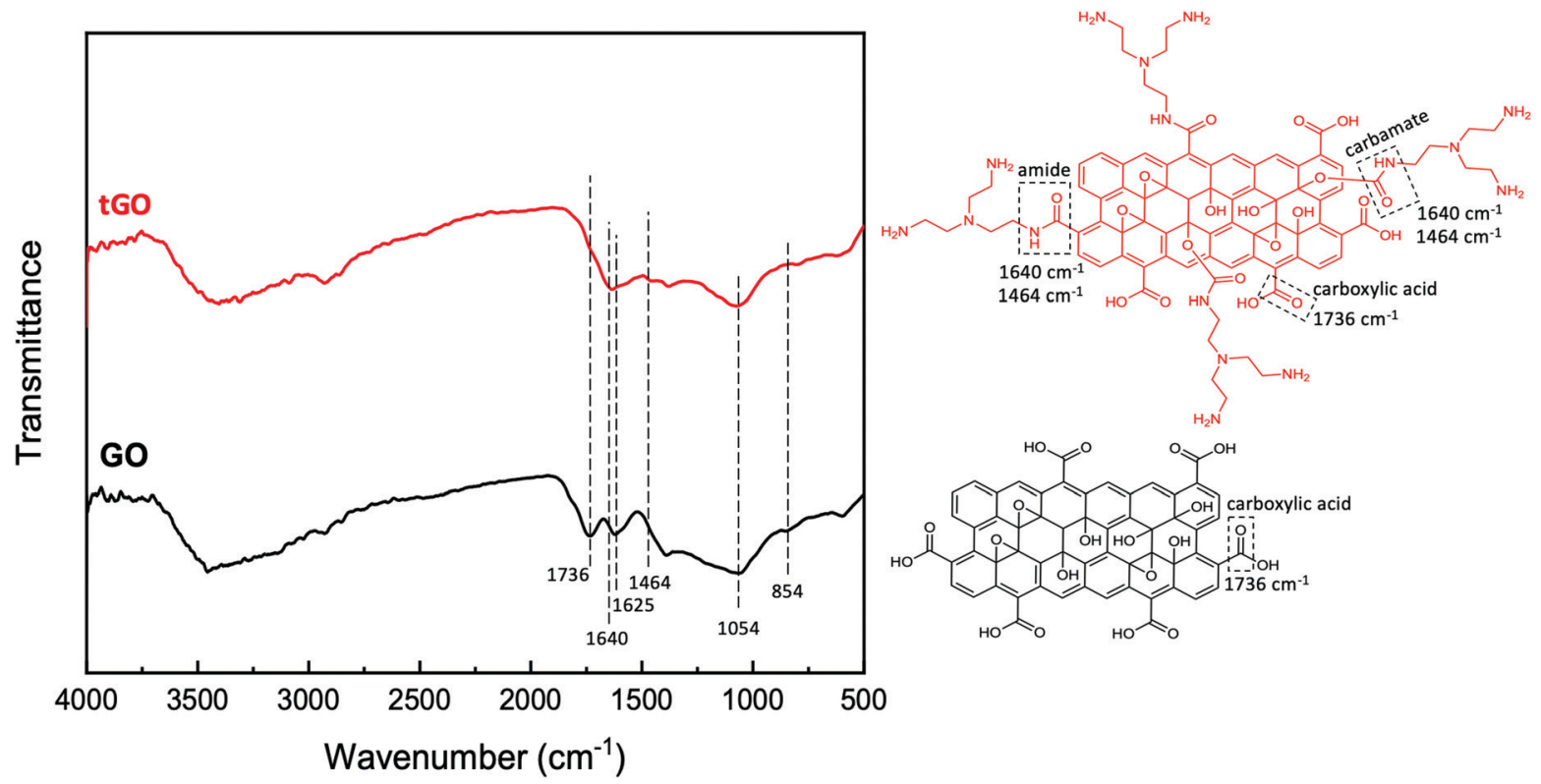

Fig. 3 FTIR analysis of native GO and tGO particles and corresponding model structures.

bonds have the same FTIR signature and the adsorption of air moisture by tGO prevents the quantification of the hydroxyl groups converted in carbamate bonds, ${ }^{100}$ it is not possible to quantitatively distinguish the two conjugation routes. Finally, the persistence of the epoxy peak at $\sim 850 \mathrm{~cm}^{-1}$ in the tGO sample indicates that TREN reaction to epoxide groups is unlikely.

Experimental and in silico evaluation of DOX adsorption on tGO. The loading of DOX on the ensemble of tGO particles was evaluated at four conditions, namely low salinity at different $\mathrm{pH}(4,6$, and 9) and PBS at $\mathrm{pH} 7.4$. The adoption of solutions at low ionic strength and different $\mathrm{pH}$ was aimed to study the effect of surface charge ( $\zeta$ potential) alone on DOX loading. The comparison between neutral buffers with different ionic strengths $(1 \mu \mathrm{M}$ at pH 6 vs. $10 \mathrm{mM}$ PBS at pH 7.4) was intended to investigate the effect of ionic strength, which both shields electrostatic repulsion and promotes mild hydrophobic interactions (sodium chloride is an intermediate salt in the Hofmeister series). ${ }^{101}$ The resulting values of DOX loading, collated in Fig. 4A and Table S1 (ESI $\dagger$ ), indicated that under acidic condition native $\mathrm{GO}(\zeta$ potential $=-36.5 \mathrm{mV})$ gave the
(A)

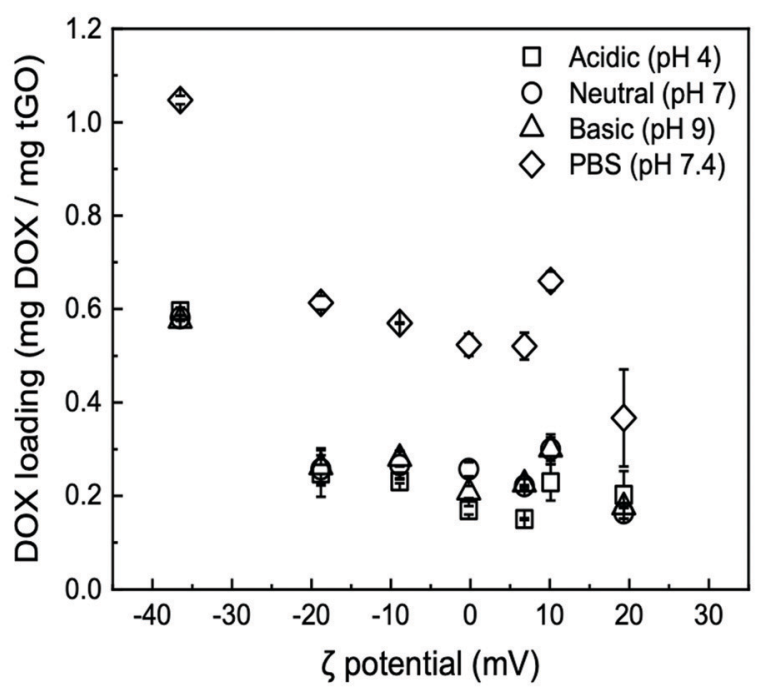

(B)

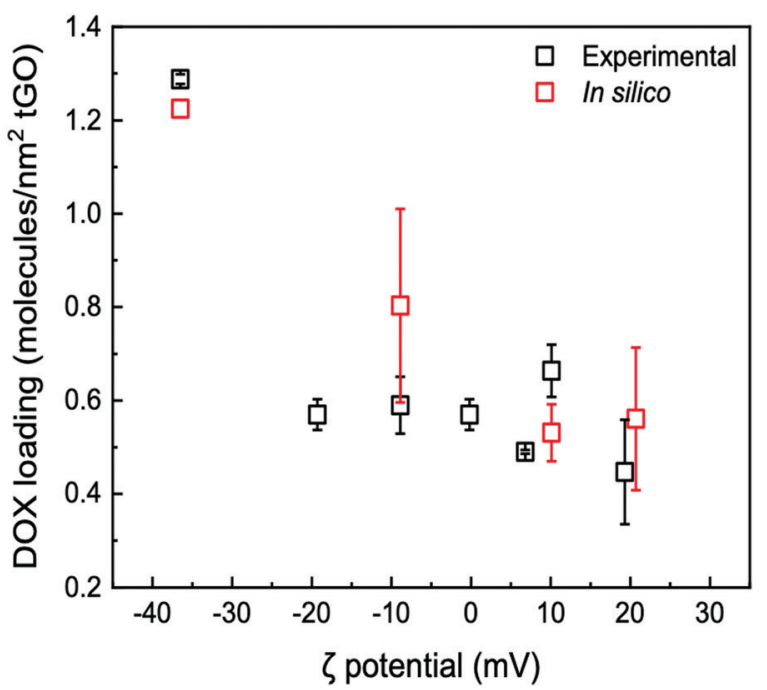

Fig. 4 (A) Loading of DOX onto $\mathrm{tGO}$ particles as a function of $\zeta$ potential at different loading conditions: Milli- $\mathrm{Q}$ water (titrated with $\mathrm{HCl}$ or $\mathrm{NaOH}$ ) at $\mathrm{pH} 4$ (squares), pH 6 (circles), and pH 9 (triangles), and PBS at pH 7.4 (diamonds), where error bars represent mean $\pm 99 \% \mathrm{Cl}(n \geq 3) ;(B) \mathrm{comparison}$ between the values of DOX loading measured at pH 6 (black) vs. predicted by the MD simulations at $\mathrm{pH} 6.1$ (red). The experimental error is presented as mean \pm $99 \% \mathrm{Cl}(n \geq 3)$, while the error from the simulations is presented as mean \pm S.D. for $n \geq 4$ independent simulations with distinct initial configurations. 
highest loading at $0.595 \pm 0.008 \mathrm{mg} \mathrm{mg}^{-1}$ GO. However, as $\zeta$ potential increases to $-18.8 \mathrm{mV}(0.449 \mu \mathrm{mol}$ TREN per $\mathrm{mg}$ of GO), the loading of DOX undergoes a sharp decline to $0.248 \pm$ $0.050 \mathrm{mg} \mathrm{mg}^{-1} \mathrm{GO}$. As $\zeta$ potential increases to $-8.9 \mathrm{mV}$ $\left(0.592 \mu \mathrm{mol} \mathrm{mg}{ }^{-1}\right)$, the loading of DOX remains constant at $0.231 \pm 0.004 \mathrm{mg} \mathrm{mg}^{-1} \mathrm{GO}$. As $\zeta$ potential further increases to $0 \mathrm{mV}$ and $6.8 \mathrm{mV}$, the loading of DOX decreases only slightly to $0.169 \pm 0.009 \mathrm{mg} \mathrm{mg}^{-1} \mathrm{GO}$ and $0.150 \pm 0.002 \mathrm{mg} \mathrm{mg}^{-1} \mathrm{GO}$, respectively. Lastly, the loading at $\zeta$ potential $=19.3 \mathrm{mV}$ $\left(0.789 \mu \mathrm{mol} \mathrm{mg}{ }^{-1}\right)$ was $0.202 \pm 0.051 \mathrm{mg} \mathrm{mg}^{-1} \mathrm{GO}$.

At low ionic strength, DOX loading at neutral and basic $\mathrm{pH}$ displays the same downward trend, featuring an initial sharp decrease from $0.581 \pm 0.004$ and $0.576 \pm 0.011 \mathrm{mg} \mathrm{mg}^{-1} \mathrm{GO}$ $(\zeta$ potential $=-36.5 \mathrm{mV})$ to $0.257 \pm 0.030 \mathrm{mg} \mathrm{mg}^{-1} \mathrm{GO}$ and $0.262 \pm 0.040$ ( $\zeta$ potential $=-18.8 \mathrm{mV}$ ) followed by a shallowed decrease, reaching $0.163 \pm 0.012 \mathrm{mg} \mathrm{mg}^{-1} \mathrm{GO}$ and $0.176 \pm 0.002$ at $\zeta$ potential $=19.3 \mathrm{mV}$. At the same time, a subtle yet statistically significant increase in DOX loading at neutral and basic $\mathrm{pH}$ vs. acidic $\mathrm{pH}$ is observed on the tGO samples with $\zeta$ potential between $-10 \mathrm{mV}$ and $+10 \mathrm{mV}$. As the $\mathrm{pH}$ of the aqueous phase increases, in fact, the positive charge carried by $\mathrm{DOX}^{83}$ and TREN is softened, and consequently DOX loading is improved.

DOX loading conducted at higher ionic strength (PBS, $\mathrm{pH}$ 7.4) exhibited the same trend observed in the low salt concentration regimes, but consistently at a higher magnitude. Specifically, the loading of DOX on native GO nearly doubles, reaching $1.048 \pm 0.009 \mathrm{mg} \mathrm{mg}^{-1} \mathrm{GO}$; on tGO samples with higher $\zeta$ potential, DOX loading decreases to within a range of values between $0.613 \pm 0.015 \mathrm{mg} \mathrm{mg}^{-1} \mathrm{GO}$ and $0.367 \pm$ $0.104 \mathrm{mg} \mathrm{mg}^{-1} \mathrm{GO}$.

These results collectively indicate two prominent trends. First, as the $\zeta$ potential of tGO increases with the modification level, the electrostatic repulsion inhibits the adsorption of DOX molecules onto the surface of tGO. Second, increasing the ionic strength of the loading solution partially screens DOX/tGO electrostatic repulsion and concurrently promotes DOX/tGO hydrophobic interactions, resulting in a constant upward shift in DOX loading across the entire range of TREN modification.

To visualize these phenomena at the molecular level, we performed a number of molecular dynamic (MD) simulations of DOX adsorption onto tGO model surfaces constructed by appending TREN moieties onto a flat, two-faced $4.8 \mathrm{~nm} \times$ $5.1 \mathrm{~nm}$ GO surface. Coherently with the FTIR results, the TREN moieties were appended through carbamate bonds to the hydroxyl groups on the GO surface. All MD simulations were performed at nearly neutral $\mathrm{pH}$ and in absence of salt, since the modeling of buffered aqueous systems is computationally complex and outside the scope of this work. ${ }^{102-104}$ As expected, the values of DOX loading predicted by the MD simulations describe a downward trend with $\zeta$ potential (Fig. 4B), caused by the electrostatic repulsion between the positively charged daunosamine moiety of DOX and the TREN moieties on tGO. On the other hand, the spacing between TREN moieties on the tGO surface is sufficient to allow adsorption of DOX molecules on surface patches of native graphene oxide (Fig. S4, ESI $\dagger$ ) across the entire range of degree of modification (0.3590.491 TREN molecules $\mathrm{nm}^{-2}$ of tGO surface).

Notably, the values of DOX loading measured experimentally and those obtained in silico show excellent quantitative agreement (Fig. 4B), indicating that the proposed in silico model portrays accurately the mechanism of DOX adsorption onto tGO particles. The simulations also indicate the formation of aggregated stacks of DOX molecules onto the tGO surface at the binding equilibrium (Fig. S4, ESI $\dagger$ ); the presence of aggregate aggregates will be functional to formulate a mechanism explaining the experimental data of DOX release presented subsequently.

Evaluation of DOX release from tGO. Studies of DOX release from GO and tGO particles were performed at $\mathrm{pH} 5$ and $\mathrm{pH} 7.4$ to simulate cancerous and healthy cellular environments, respectively. The release profiles obtained at $\mathrm{pH} 5$ are reported in Fig. 5, while those obtained at $\mathrm{pH} 7.4$ are reported in Fig. S5 (ESI $\dagger$ ).

Two time scales are of relevance in this study: (i) short term (72 h), which is representative of a typical therapeutic window for in vitro DDS validation, and (ii) long term $(>600 \mathrm{~h})$, which fits the time scale of maintenance chemotherapy. The values of DOX percent release from GO and tGO particles at both $\mathrm{pH} 5$ and 7.4 for 72 and $650 \mathrm{~h}$ are reported in Tables S2 and S3 (ESI $\dagger$ ), respectively. As anticipated, the release experiments conducted at pH 5 afforded higher values of DOX percent release compared to those at $\mathrm{pH}$ 7.4. The lower $\mathrm{pH}$ promotes the positive charge on both DOX and TREN moieties, which causes DOXDOX and DOX-TREN repulsion thereby triggering release. Because drug release in a tumor-mimetic environment is more relevant in the context of this work, our analysis focuses on the release data generated at $\mathrm{pH}$ 5. The comparison of DOX release from the particles loaded at low ionic strength shows that only two tGO particles outperform the native GO particles, namely tGO with $\zeta$ potential $=-18.8 \mathrm{mV}$ and $-8.9 \mathrm{mV}$. The former afforded a 72 h-release of $13.7 \pm 2.1 \%$ (when loaded $\mathrm{pH} 4$ ), $12.4 \pm 0.4 \%(\mathrm{pH} 6)$, and $11.4 \pm 0.8 \%(\mathrm{pH} 9)$, and a 650 h-release of $22.7 \pm 3.2 \%(\mathrm{pH} 4), 19.2 \pm 4.2 \%(\mathrm{pH} 6)$, and $19.8 \pm 4.0 \%$ $(\mathrm{pH} 9)$. The latter showed a 72 h-release of $9.0 \pm 1.1 \%(\mathrm{pH} 4)$, $9.2 \pm 0.3 \%(\mathrm{pH} 6)$, and $10.6 \pm 1.5 \%(\mathrm{pH} 9)$, and a 650 h-release of $16.7 \pm 4.0 \%(\mathrm{pH} 4), 15.7 \pm 3.8 \%(\mathrm{pH} 6)$, and $19.8 \pm 3.9 \%$ (pH 9). Comparatively, the native GO released only $\sim 6 \%$ DOX after $72 \mathrm{~h}$ and $\sim 15 \%$ after $650 \mathrm{~h}$. On the other hand, tGO carriers with higher $\zeta$ potential afforded a lower release of DOX.

Given the negligible dependence of DOX loading upon $\mathrm{pH}$ at low salt concentration as well as DOX release from the resulting particles, only the DOX/GO systems loaded at neutral $\mathrm{pH}$ (low vs. high salt concentrations) were considered in the rest of this study. The values of DOX percent release at $\mathrm{pH} 5$ for $72 \mathrm{~h}$ and $650 \mathrm{~h}$ plotted against the values of $\zeta$ potential of the corresponding GO and tGO particle carriers are presented in Fig. 6A and B, respectively; the analogous plots for release at $\mathrm{pH}$ 7.4 are presented in Fig. S6 (ESI $\dagger$ ).

It is immediately evident that DOX release is determined by two parameters, namely (i) the ionic strength of DOX solution during loading and (ii) the $\zeta$ potential of the particles.

First, regarding the ionic strength of the loading solution, a significant increase is observed in the values of DOX percent 
(A)

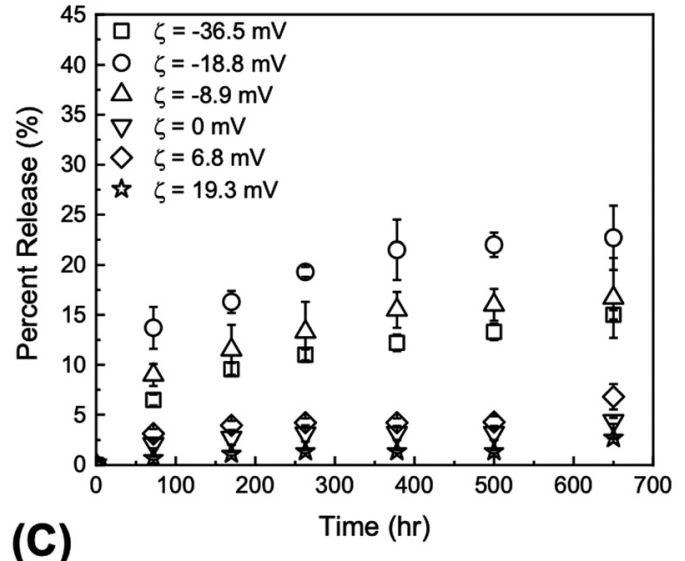

(C)

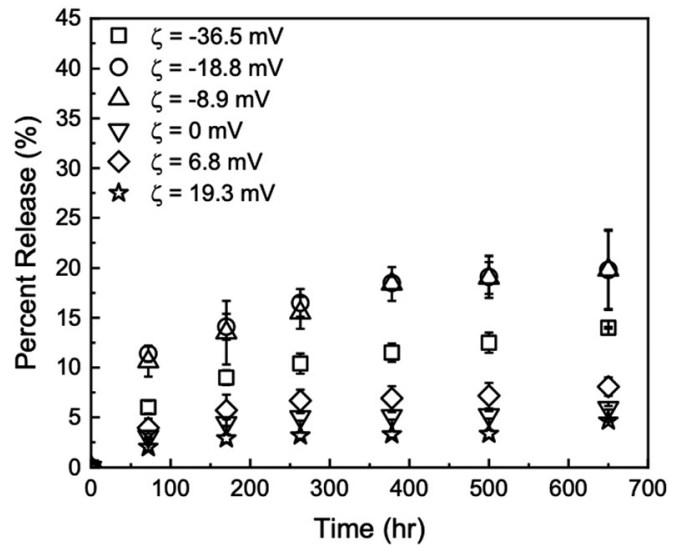

(B)
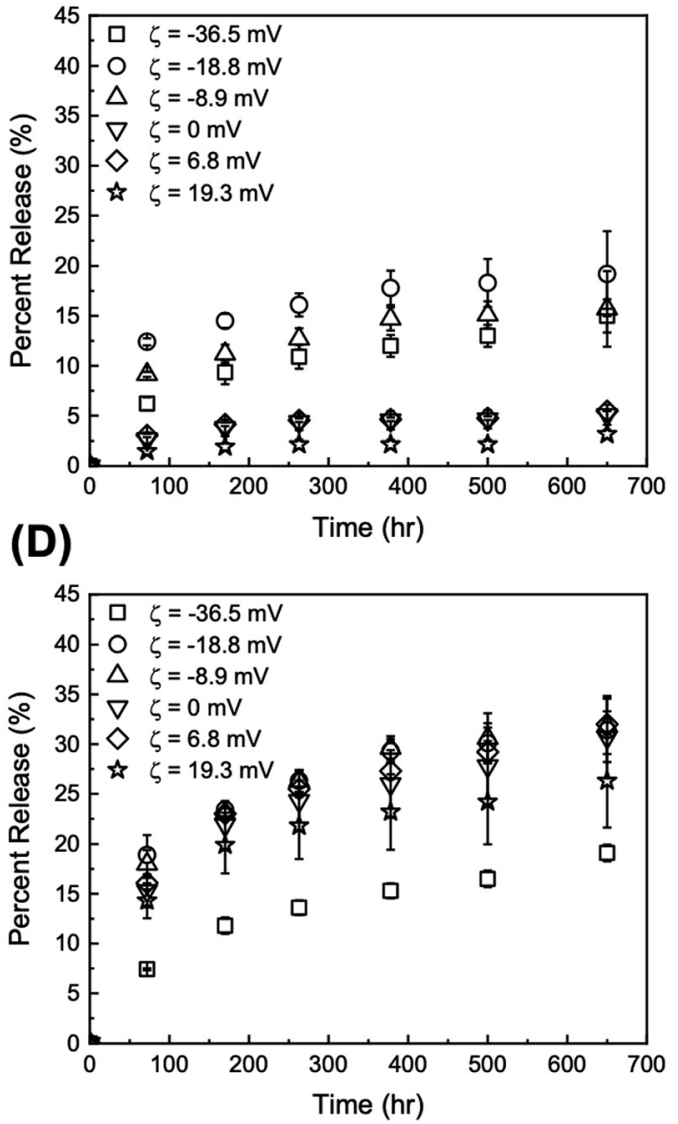

Fig. 5 Release profiles at $\mathrm{pH} 5$ from DOX/tGO particles loaded at (A) $\mathrm{pH} 4$, (B) $\mathrm{pH}$ 6, (C) $\mathrm{pH}$ 9, and (D) PBS at pH 7.4. Error bars represent mean $\pm 99 \% \mathrm{Cl}(n \geq 3)$.

(A)

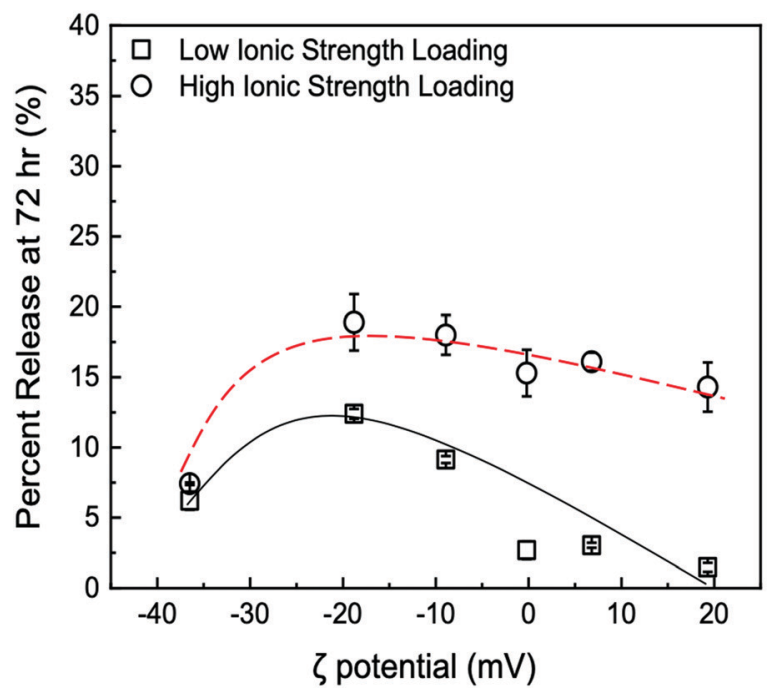

(B)

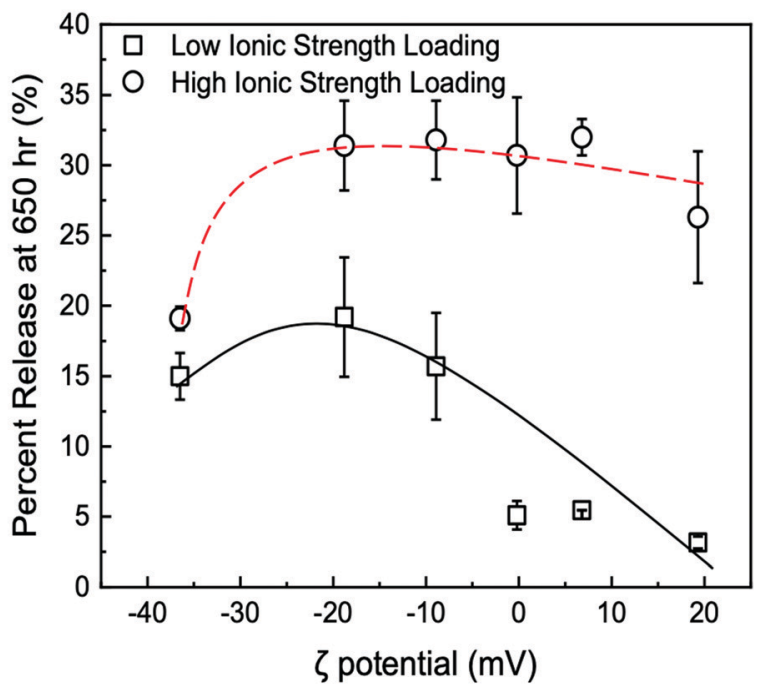

Fig. 6 Values of DOX percent release at $\mathrm{pH} 5$ after (A) $72 \mathrm{~h}$ and (B) $650 \mathrm{~h}$ from DOX/GO and DOX/tGO particles loaded at neutral pH and low ionic strength (squares) or high ionic strength (circles). Hashed are added to guide the visualization of the results. Error bars represent mean $\pm 99 \% \mathrm{Cl}(n \geq 3$ ).

release, which shift from a range of 1.48-12.4\% (low salt and from 3.16-19.2\% (low salt loading) to 19.1-31.8\% (high salt loading) to $7.42-18.9 \%$ (high salt loading) for $72 \mathrm{~h}$ release, loading) for $650 \mathrm{~h}$ release. In this regard, the in silico modeling 
of DOX adsorption indicated the formation of DOX aggregates both in solution and onto the GO surface. The extent of aggregation is likely enhanced at high salt conditions, where electrostatic repulsion is softened and hydrophobic interactions are promoted, as observed with drug-like molecules; ${ }^{105}$ accordingly, we hypothesize that DOX adsorbs onto the tGO surface in a predominantly mono-/di-meric form when loaded in a low ionic strength environment, and in a rather multimeric form at higher ionic strength. When exposed to $\mathrm{pH}$ 5, DOX aggregates are considerably less stable than monomeric DOX, due to the combination of DOX-DOX and DOX-TREN electrostatic repulsion, and are released more easily, resulting in an upward shift in percent release.

The dependence of DOX percent release on $\zeta$ potential, on the other hand, is rather counterintuitive. In place of a monotonic trend reverse to that of DOX loading (Fig. 4B), in fact, concave downward curves were obtained (Fig. 6A and B). To explain this behavior, one must consider the charge environment onto the GO particles and in the layer of charges surrounding them (Fig. 7). The surface of GO is inherently negatively charged, due to the numerous oxygen-containing moieties; ${ }^{106}$ on this surface, the TREN moieties and the DOX molecules form a layer of "condensed" positive counter-ions (Stern layer), respectively covalently linked and non-covalently adsorbed. The enveloping Gouy layer, framed by the Stern plane and the slipping plane, at which the $\zeta$ potential is measured, contains a mixture of positive (e.g., $\mathrm{H}^{+}$and $\mathrm{Na}^{+}$) and negative (e.g., $\mathrm{OH}^{-}$and $\mathrm{Cl}^{-}$) counter-ions. On virgin $\mathrm{GO}$,
DOX molecules adsorbed at $\mathrm{pH} 6$ are strongly retained by the negatively charged moieties on the surface of GO (Fig. 7A); as the environment surrounding the DOX/GO particles is adjusted to $\mathrm{pH} 5$, the softening of the inherent negative charge on the surface of GO and the DOX-DOX electrostatic repulsion molecules triggers release. On tGO particles with low TREN modification ( $\zeta$ potential $<0$ ), DOX molecules are adsorbed on patches of native GO between TREN moieties (Fig. 7B); at pH 5, the softening of the negative charge on GO, and the combined DOX-DOX and DOX-TREN repulsion favors DOX desorption, resulting in an increment of percent release. On tGO particles with high TREN modification ( $\zeta$ potential $\approx$ or $\geq 0$ ), the higher density of TREN moieties in the Stern layer drives the accumulation of negative $\left(\mathrm{OH}^{-}\right.$and $\left.\mathrm{Cl}^{-}\right)$counter-ions within the Gouy layer, resulting in the electric stabilization of the cationic DOX molecules adsorbed onto the surface of GO (note the cyan spheres representing $\mathrm{Cl}^{-}$ions intercalated between DOX and TREN molecules in Fig. 7C and D); this results in a softening of DOX-TREN repulsions at $\mathrm{pH}$ 5, which translates in a decrease of percent release. These phenomena are evident when DOX loading is performed at low ionic strength. When DOX loading is operated in PBS, in fact, the TREN moieties are stabilized by counter-anions prior to DOX adsorption and DOX release at pH 5 is mostly triggered by the disassembly of drug aggregates by DOX-DOX electrostatic repulsion. This makes the values of percent release much less dependent upon $\zeta$ potential. Native GO is an exception, given its complete lack of TREN modification.
(A)

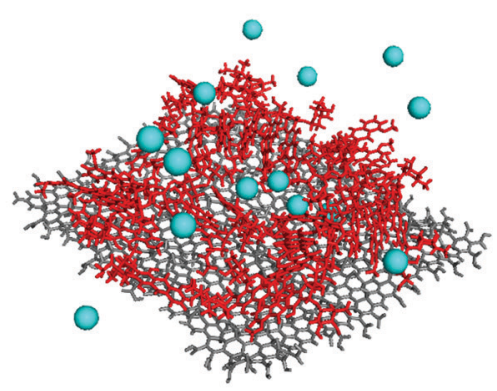

(C)

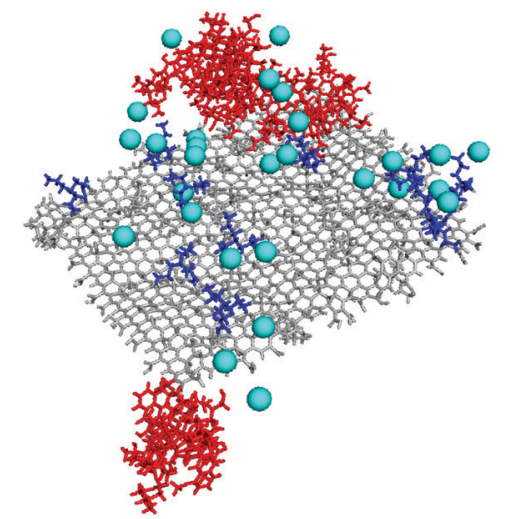

(B)

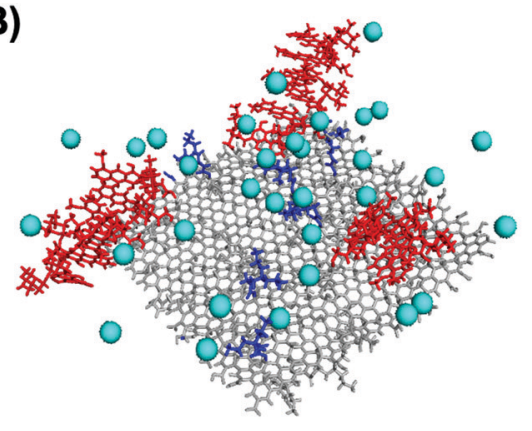

(D)

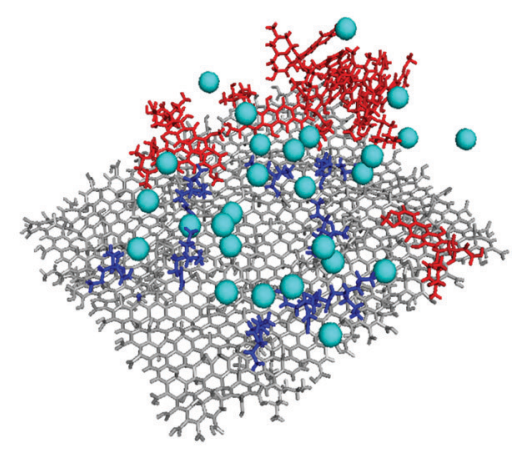

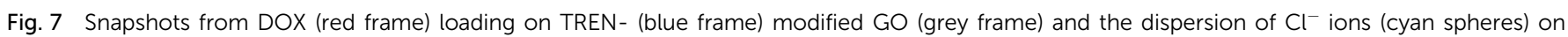
(A) native GO $(\zeta=-36.5 \mathrm{mV})$, (B) tGO $(\zeta=-8.9 \mathrm{mV}),(\mathrm{C})$ tGO $(\zeta=10.1 \mathrm{mV})$, and (D) tGO $(\zeta=20.7 \mathrm{mV})$. 


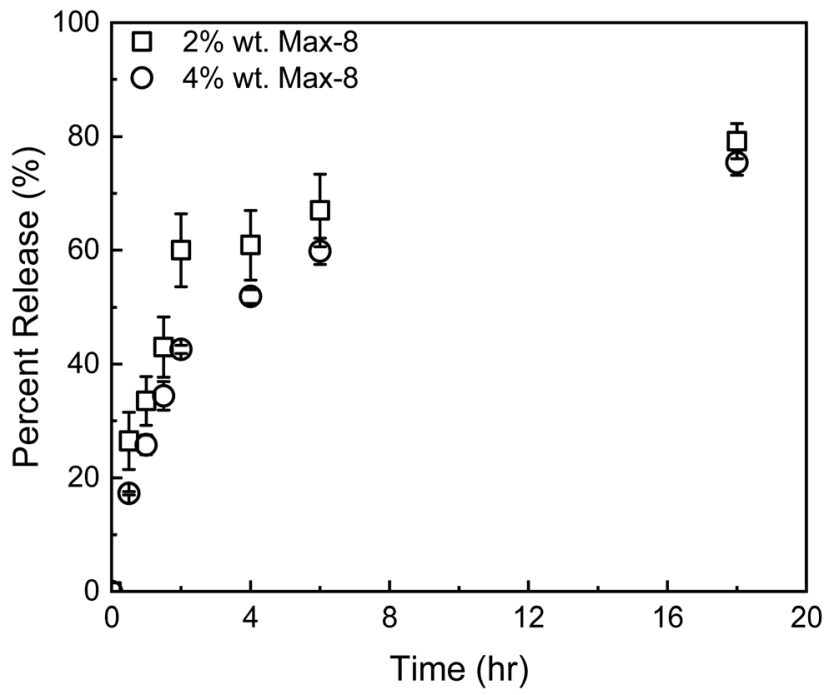

Fig. 8 Release profiles of GEM from a Max8 hydrogel at $2 \mathrm{w} / \mathrm{w}$ (squares) and $4 \mathrm{w} / \mathrm{w}$ (circles). Error bars represent mean $\pm 99 \% \mathrm{Cl}(n \geq 3)$.

\section{Synthesis and characterization of tGO-Max8 composite}

Release of GEM and DOX from tGO-Max8 composite. Based on the results of the DOX release studies, we selected tGO particles with $\zeta$ potential of $-18.8 \mathrm{mV}$ as model carrier to develop the composite drug delivery system (DDS); the high loading of DOX attained in PBS at $\mathrm{pH} 7.4$ enables reducing the amount of tGO particles required to achieve therapeutic efficacy, which minimizes the risk of cytotoxic effects. To develop the hydrogel matrix, we initially sought to determine the optimal peptide concentration that affords rapid gelation and yields a homogenous gel that poses no diffusion limitations to the transport of either GEM or DOX. Peptide gel fractions of 2 and $4 \mathrm{w} / \mathrm{w}$ were chosen owing to their nearly instant gelation kinetics. The release kinetics from GEM-loaded gels are presented in Fig. 8. Within the first 4 hours, the $2 \mathrm{w} / \mathrm{w}$ hydrogel releases GEM more rapidly, reaching $60.9 \pm 6.1 \%$ compared to $51.9 \pm 1.2 \%$ by the $4 \mathrm{w} / \mathrm{w}$ hydrogel. After $16 \mathrm{~h}$, however, the amount of GEM released is independent of gel fraction, reaching $\sim 80 \%$. As we envision a scheduled/sequential delivery system wherein the first drug in the chemotherapy regimen (GEM) is released more rapidly, we elected to use the $2 \mathrm{w} / \mathrm{w}$ hydrogel as the matrix for embedding the DOX/tGO particles.

The composite DDS was prepared by dissolving Max8 peptide at $2 \mathrm{w} / \mathrm{w}$ in a sonicated aqueous suspension of the selected DOX/tGO particles. The instantaneous formation of the peptide hydrogel resulted in a homogeneous dispersion of the particles. Confocal fluorescence microscopy images of the control tGO-Max8 composite (Fig. S7, ESI $\dagger$ ) and DOX/tGOMax8 composite immediately after gel formation (Fig. S7, ESI $\dagger$ ) confirms the homogeneous dispersion of the tGO particles; further, Fig. S7 (ESI $\dagger$ ) indicates DOX remains bound to the tGO particles upon gel formation.

The release kinetics of the composite tGO-Max8 systems are presented in Fig. 9. It is first noted that the peptide hydrogel poses a notable resistance to the transport of DOX, resulting in a 3 -fold reduction of the percent release in the aqueous phase surrounding the hydrogel, from the $19.0 \pm 0.1 \% 72$ h-release given by free DOX/tGO particles in solution to the $6.2 \pm 0.2 \%$ given by DOX/tGO particles embedded in the Max8 scaffold. This is likely caused by the cationic and amphiphilic nature of the Max8 peptide (rich in positively charged lysine and hydrophobic valine residues), which limits the partitioning of DOX - a positively charged molecule - from the tGO surface to
(A)

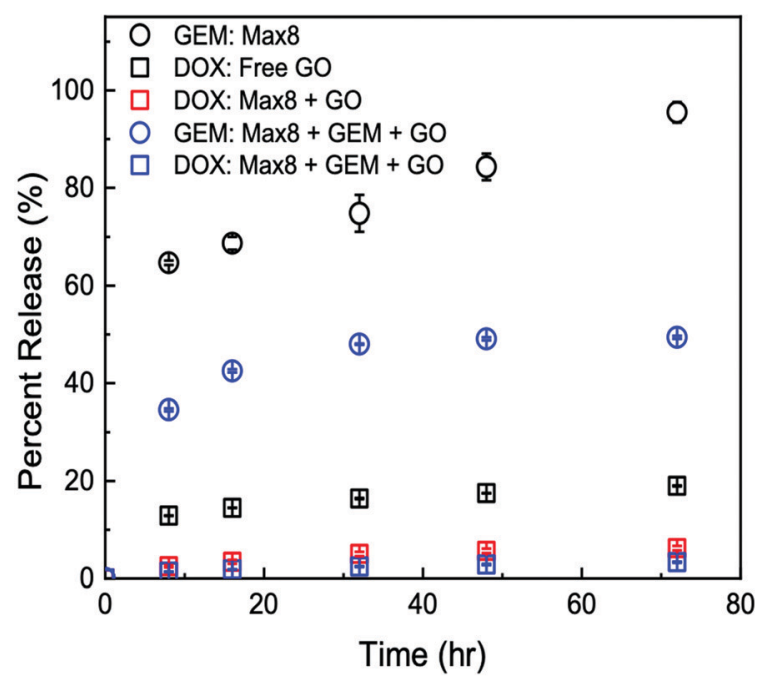

(B)

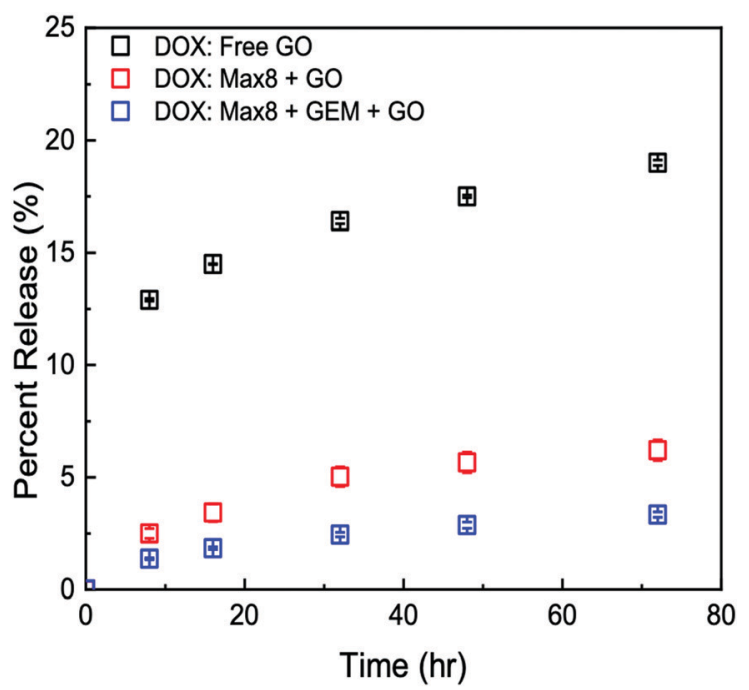

Fig. 9 (A) Release profiles of GEM from 2 w/w GEM/Max8 (black circles), DOX from free DOX/tGO particles (black squares), DOX from DOX/tGO particles embedded in a 2 w/w Max8 hydrogel (red squares), GEM from a 2 w/w GEM/Max8 hydrogel loaded with tGO particles (blue circles), and DOX from DOX/tGO particles embedded in a 2 w/w GEM/Max8 hydrogel (blue); (B) release of DOX from the system listed in panel (A). Error bars represent mean $\pm 99 \% \mathrm{Cl}(n \geq 3)$. 
the hydrogel phase and its migration through the hydrogel matrix, as noted in prior work. ${ }^{107}$ We have observed that the display of hydrophobic moieties in polycationic hydrogels hinders the migration of DOX. Second, we observed a drastic change in GEM release after $72 \mathrm{~h}$, from $95.5 \pm 1.1 \%$ from the GEM/Max8 hydrogel alone compared to $49.4 \pm 0.2 \%$ from the composite system. This is likely caused by the interaction between GEM and the DOX/tGO nanoparticles during its diffusion pathway. Finally, the concurrent migration of the two drugs results in a further decrease of DOX release, from the $6.2 \pm 0.2 \%$ given by the GEM-free composite to the $3.3 \pm 0.1 \%$ given by the GEM-loaded hydrogel after $72 \mathrm{~h}$. This is attributed to DOX-GEM interactions that have been documented to occur and drastically change diffusion properties of the DOX-GEM pair through poly-cationic hydrogel matrices. ${ }^{107}$

\section{In vitro characterization}

To determine the efficacy of the composite system relative to free single drug and drug combination treatments, we conducted in vitro evaluation of drug-loaded DOX/tGO-GEM/Max8 composites using the triple negative breast cancer cell line MDA-MB-231. The results are presented as dose-response curves in Fig. 10A, fit with the median-effect equation. ${ }^{108}$ The corresponding IC $_{50}$ values are presented in Fig. 10D. Free DOX and free GEM yielded $\mathrm{IC}_{50}$ values of $0.399 \pm 0.04 \mu \mathrm{M}$ and $>20 \mu \mathrm{M}$, respectively, in line with published data. ${ }^{9}$

In testing the free DOX/tGO particles, we referred to the values of $72 \mathrm{~h}$-release at $\mathrm{pH} 5$ to determine the concentration of particles affording a release of DOX at a dose equivalent to that utilized in the free drug study and accounted for the possibility of uptake of the DOX/tGO particles. The range of DOX concentration utilized in this study, 0.025-1.6 $\mu \mathrm{M}$, translated in a dose of $0.2-14.2 \mu \mathrm{g}$ tGO $\mathrm{mL}^{-1}$ of total release volume. The same volume of drug-free particles was also tested as a control to evaluate their basal cytotoxicity (Fig. 10C). Notably, the DOX/tGO particles featured a cytotoxic activity comparable to that of free DOX, with an $\mathrm{IC}_{50}$ value of $0.131 \pm 0.002 \mu \mathrm{M}$, corresponding to $\sim 1.7 \mu \mathrm{g}$ of DOX/tGO particles $\mathrm{mL}^{-1}$ (Fig. 10B). At the same time, the tGO particles alone proved relatively biocompatible, yielding a cell survival rate above $90 \%$ when employed at concentrations below $14.2 \mu \mathrm{g} \mathrm{mL}{ }^{-1}$. Most importantly, at the dose of $1.7 \mu \mathrm{g} \mathrm{mL} \mathrm{m}^{-1}$ needed for the DOX-loaded particles to match the $\mathrm{IC}_{50}$ of free DOX, the tGO particles alone show a cell survival rate of $\sim 99 \%$.

The cytotoxicity of the DOX-GEM combination was then evaluated at the reference GEM : DOX molar ratio for of $10: 1$, reported as synergistic in prior work (Fig. 10D). ${ }^{9}$ For the free-drug combination, we observed an $\mathrm{IC}_{50}$ value of $0.135 \pm$ $0.035 \mu \mathrm{M}$, relative to DOX concentration. This corresponded to a combination index (CI), calculated utilizing the Chou-Talalay

\section{(A)}

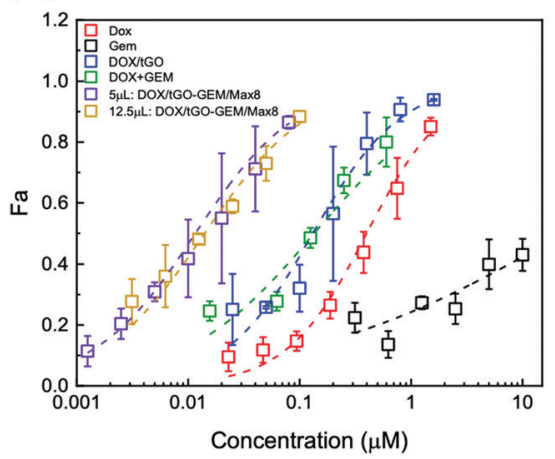

(C)

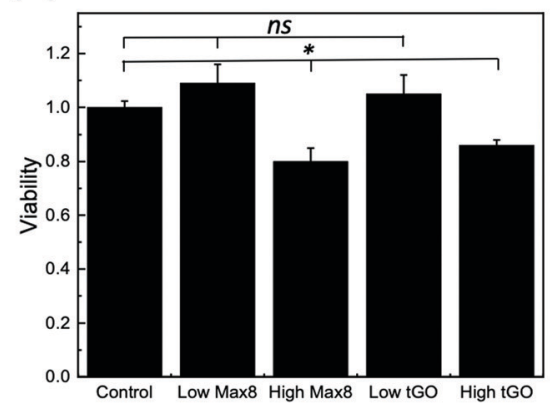

(B)

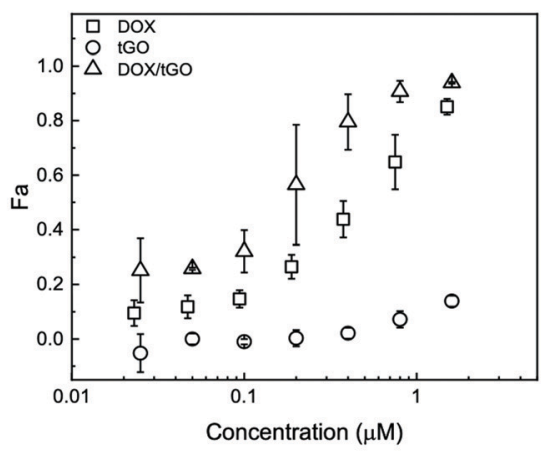

(D)

\begin{tabular}{|c|c|c|}
\hline Formulation & IC $_{\mathbf{5 0}}$ Conc. $(\boldsymbol{\mu M})$ & $\mathbf{C I}$ \\
\hline DOX & $0.399 \pm 0.04$ & $\mathrm{~N} / \mathrm{A}$ \\
\hline GEM & $>20$ & $\mathrm{~N} / \mathrm{A}$ \\
\hline tGO/DOX & $0.132 \pm 0.002$ & $\mathrm{~N} / \mathrm{A}$ \\
\hline DOX + GEM & $0.135 \pm 0.035$ & $0.396 \pm 0.035$ \\
\hline $5 \mu$ L Composite & $0.012 \pm 0.0004$ & $0.093 \pm 0.001$ \\
\hline $12.5 \mu$ L Composite & $0.014 \pm 0.001$ & $0.113 \pm 0.002$ \\
\hline
\end{tabular}

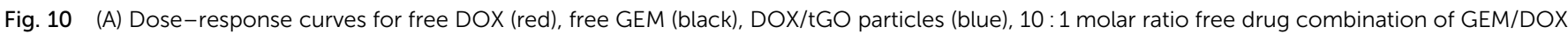

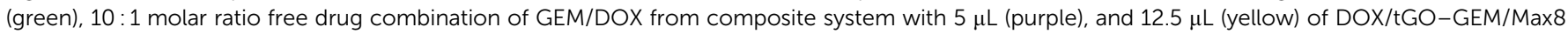

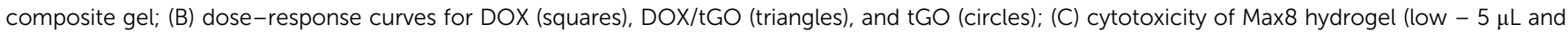

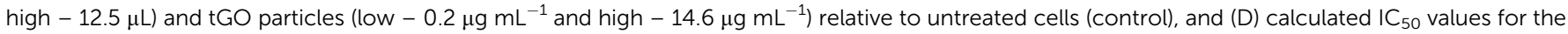

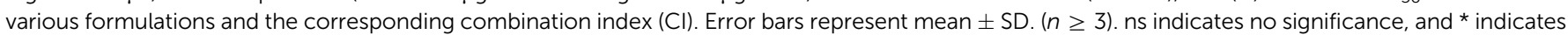
$p<0.05$, as obtained from a Student's $t$-test. 
method, ${ }^{109}$ of $0.396 \pm 0.035$, confirming strong synergism of the drug pair, consistent with that obtained by Vogus et al. ${ }^{9}$ using the same drug pair and concentration, and cancer cell line. Lastly, we sought to demonstrate that the dual-drug loaded tGO-Max8 composite system is more therapeutically efficacious than the free drug combination. Accordingly, the reference 10:1, GEM:DOX molar ratio was utilized in these release experiments. We observed an $\mathrm{IC}_{50}$ value of $0.0116 \pm$ $0.0004 \mu \mathrm{M}$, relative to DOX concentration, corresponding to a $\mathrm{CI}$ of $0.093 \pm 0.001$, indicating a stronger synergism than the free drug pair. It is noted that the reported values of CI are comparable to those obtained by Vogus et al. of $\mathrm{CI}=\sim 0.12$ with sequential administration of this drug pair (GEM for $24 \mathrm{~h}$, then DOX for $48 \mathrm{~h}) .^{9}$ We attribute this further reduction in CI (higher synergism) to the ability of the composite tGO-Max8 system to control the release kinetics and achieve the postulated scheduled release, known to improve synergism. ${ }^{9,10}$ Notably, the $\mathrm{IC}_{50}$ value of the composite system corresponds to a dose of $16 \mu \mathrm{g} \mathrm{mL} \mathrm{m}^{-1}$ of tGO particles and $5 \mu \mathrm{L}$ of Max8 hydrogel, both proven to be non-cytotoxic per se (Fig. 10C).

\section{Conclusions}

Scheduled delivery of synergistic combinations of chemotherapeutics is increasingly regarded as a highly effective treatment for aggressive solid tumors. The precise control of molar ratio, sequence, and rate of delivery, in fact, enhances drug synergism and enables a drastic reduction of the required therapeutic doses, while maintaining a highly efficacious outcome. To date, two main approaches to achieve scheduled delivery appear in clinical settings: portable devices that accompany the patient through therapy and inject different drugs based on a set timetable, or engineered materials featuring fine-tuned drug release kinetics. The design and optimization of the chemical functionalization and organization of composite materials as drug delivery systems (DDS) greatly benefits from the integration of in silico design and experimental evaluation. By providing a deeper insight into the complex physicochemical interactions between drugs and materials, integrated computational-experimental methods enhance the quality and accelerate the process of discovery and validation of therapeutic products. This study aims to apply this concept to the design of a homogenous hydrogel-nanoparticle composite capable of delivering a recognized synergistic drug pair (DOX and GEM) with precise molar ratio and release kinetics. In this context, we resolved to (i) adopt materials that combine biocompatibility and affordability with chemically versatility, and (ii) implement scalable functionalization strategies to ensure the translational potential of the resulting DDS. GO nanoparticles and Max8 peptide hydrogel fulfill the requirements on the materials, while TREN modification of the GO surface and the tuning of the Max8/water ratio represent a scalable approach to achieve precise control over the ratio and kinetics of drug delivery. Crucial to the design and understanding of the proposed DDS has been the development of an MD model capable of describing quantitatively the DOX loading on and release from the modified GO particles. The homogeneous distribution of DOX-tGO particles and the thixotropic nature of the Max8 hydrogel matrix make the proposed formulation an exquisitely injectable one, ideal for the treatment of solid tumors through both first-line or consolidation chemotherapy. In this regard, the MDA-MB-231 triple negative breast cancer (TNBC) cell line adopted in this work represents an ideal target for the preclinical testing of the proposed DDS. Patients with metastatic TNBC, in fact, have a poor prognosis and a median overall survival of $\sim 13$ months upon treatment. In the attempt to contribute to the development of a therapeutic arsenal against TNBC, we have tailored our composite system to release GEM at 47 -fold rate and a 10-fold molar ratio compared to DOX, which the literature indicates as highly synergistic. The results presented herein demonstrate the validity of our design: the composite DDS afforded a remarkable combination index of $0.093 \pm 0.001$. This value is not only lower than that provided by the DOX-GEM combination as free drugs in solution (CI $=0.396 \pm 0.034)$ but is lower than any value reported in the literature for this and similar drug combinations delivered by either microfluidic devices or engineered materials. ${ }^{6,9,10,110}$ Together with reinforcing the value of combining delivery schedule and molar ratio towards increasing therapeutic synergism, this study presents a step forward in the development of translatable (effective, affordable, and scalable) solutions enabling successful treatment of unmet oncological diseases. To this end, future work on different cell lines (e.g., renal cell carcinoma Caki-2 cells or bladder carcinoma MB49 cells) will be undertaken to demonstrate the flexibility of the proposed DDS towards the treatment of different forms of cancer. Selected formulations will be characterized in vivo to evaluate, together with therapeutic efficacy, biodistribution and potential adverse effects; these, however, are not anticipated, given the biocompatibility of Max8 and the low amount of modified GO particles present in the formulation. With its focus on material design and understanding, this contribution lays the ground for the future optimization of novel composites targeting aggressive metastatic solid tumors that - to this day - impact the health and quality of life of millions of people worldwide.

\section{Author contributions}

Conceptualization, J. D. S., K. S., E. S., M. D., and S. M.; methodology, J. D. S., K. S., A. T. Y., R. L. L., E. S., M. D., and S. M.; investigation, J. D. S., K. S., A. T. Y., S. R., N. H., C. E. D., J. M. D., and R. L. L.; formal analysis, J. D. S. and K. S.; writing original draft, J. D. S., K. S., E. S., and S. M.; writing - review \& editing, J. D. S, K. S., E. S., M. D., and S. M.; funding acquisition, W. G., E. S., M. D., and S. M.; resources, N. H., W. G., E. S., M. D., and S. M.; supervision, E. S., M. D., and S. M.

\section{Conflicts of interest}

There are no conflicts to declare. 


\section{Acknowledgements}

This work was funded by start-up funds provided by NC State University. SM, SR, CED and JDS kindly acknowledge support from the Department of Education Graduate Assistance in Areas of National Need (GAANN) in Molecular Biotechnology. ATY acknowledges support from the NIH Integrative Vascular Biology Traineeship (NIH T32HL069768). KS thanks Dr. Nathan Duff and Dr. HoShin Kim for helpful discussions.

\section{Notes and references}

1 J. G. Croissant, D. Zhang, S. Alsaiari, J. Lu, L. Deng, F. Tamanoi, A. M. AlMalik, J. I. Zink and N. M. Khashab, J. Controlled Release, 2016, 229, 183-191.

2 T. Anajafi, M. D. Scott, S. You, X. Yang, Y. Choi, S. Y. Qian and S. Mallik, Bioconjugate Chem., 2016, 27, 762-771.

3 A. C. Palmer and P. K. Sorger, Cell, 2017, 171, 1678-1691.

4 K. Goto, Y. Ohe, T. Shibata, T. Seto, T. Takahashi, K. Nakagawa, H. Tanaka, K. Takeda, M. Nishio and K. Mori, et al., Lancet Oncol., 2016, 17, 1147-1157.

5 Y.-G. Lee, J. H. Lee, S.-H. Kim, Y. J. Kim, H. Lee, S. Ahn, J.-S. Jang, J.-S. Lee and J. H. Kim, Lung Cancer, 2018, 122, 88-93.

6 A. Pusuluri, V. Krishnan, D. Wu, C. W. Shields, L. W. Wang and S. Mitragotri, Bioeng. Transl. Med., 2019, 4, e10129.

7 Q. Hu, W. Sun, C. Wang and Z. Gu, Adv. Drug Delivery Rev., 2016, 98, 19-34.

8 W. Fan, B. Yung, P. Huang and X. Chen, Chem. Rev., 2017, 117, 13566-13638.

9 D. R. Vogus, A. Pusuluri, R. Chen and S. Mitragotri, Bioeng. Transl. Med., 2018, 3, 49-57.

10 D. R. Vogus, M. A. Evans, A. Pusuluri, A. Barajas, M. Zhang, V. Krishnan, M. Nowak, S. Menegatti, M. E. Helgeson and T. M. Squires, et al., J. Controlled Release, 2017, 267, 191-202.

11 Y.-X. Bao, X.-D. Zhao, H.-B. Deng, C.-L. Lu, Y. Guo, X. Lu and L.-L. Deng, Cell. Oncol., 2016, 39, 343-352.

12 Z. Zhang, S. Liu, Y. Qi, D. Zhou, Z. Xie, X. Jing, X. Chen and Y. Huang, J. Controlled Release, 2016, 235, 125-133.

13 X. Li, Y. He, J. Hou, G. Yang and S. Zhou, Small, 2019, e1902262.

14 L. Wei, J. Chen, S. Zhao, J. Ding and X. Chen, Acta Biomater., 2017, 58, 44-53.

15 H. Wang, J. Wu, K. Xie, T. Fang, C. Chen, H. Xie, L. Zhou and S. Zheng, ACS Appl. Mater. Interfaces, 2017, 9, 10567-10576.

16 S. Narayanan, U. Mony, D. K. Vijaykumar, M. Koyakutty, B. Paul-Prasanth and D. Menon, Nanomedicine, 2015, 11, 1399-1406.

17 T. Wang, D. Wang, J. Liu, B. Feng, F. Zhou, H. Zhang, L. Zhou, Q. Yin, Z. Zhang and Z. Cao, et al., Nano Lett., 2017, 17, 5429-5436.

18 L. Li, W. Sun, Z. Zhang and Y. Huang, J. Controlled Release, 2016, 232, 62-74.

19 J.-Y. Douillard, A. Sobrero, C. Carnaghi, P. Comella, E. Díaz-Rubio, A. Santoro and E. Van Cutsem, Ann. Oncol., 2003, 14, ii7-ii12.
20 L. H. J. Simkens, H. van Tinteren, A. May, A. J. ten Tije, C.-J. M. Creemers, O. J. L. Loosveld, F. E. de Jongh, F. L. G. Erdkamp, Z. Erjavec and A. M. E. van der Torren, et al., Lancet, 2015, 385, 1843-1852.

21 M. Ducreux, D. Malka, J. Mendiboure, P.-L. Etienne, P. Texereau, D. Auby, P. Rougier, M. Gasmi, M. Castaing and M. Abbas, et al., Lancet Oncol., 2011, 12, 1032-1044.

22 F. Cardoso, P. L. Bedard, E. P. Winer, O. Pagani, E. SenkusKonefka, L. J. Fallowfield, S. Kyriakides, A. Costa, T. Cufer and K. S. Albain, J. Natl. Cancer Inst., 2009, 101, 1174-1181.

23 X. Liang, C. Gao, L. Cui, S. Wang, J. Wang and Z. Dai, Adv. Mater., 2017, 29, 1703135.

24 K. M. Camacho, S. Menegatti, D. R. Vogus, A. Pusuluri, Z. Fuchs, M. Jarvis, M. Zakrewsky, M. A. Evans, R. Chen and S. Mitragotri, J. Controlled Release, 2016, 229, 154-162.

25 K. M. Camacho, S. Menegatti and S. Mitragotri, Nanomedicine, 2016, 11, 1139-1151.

26 K. M. Camacho, S. Kumar, S. Menegatti, D. R. Vogus, A. C. Anselmo and S. Mitragotri, J. Controlled Release, 2015, 210, 198-207.

27 Y. Zheng, Y. Cheng, J. Chen, J. Ding, M. Li, C. Li, J. Wang and X. Chen, ACS Appl. Mater. Interfaces, 2017, 9, 3487-3496.

28 P. S. Yavvari, S. Pal, S. Kumar, A. Kar, A. K. Awasthi, A. Naaz, A. Srivastava and A. Bajaj, ACS Biomater. Sci. Eng., 2017, 3, 3404-3413.

29 B. Balakrishnan, N. Joshi, K. Thorat, S. Kaur, R. Chandan and R. Banerjee, J. Mater. Chem. B, 2019, 7, 2920-2925.

30 Y. Wang, Q. Zhao, N. Han, L. Bai, J. Li, J. Liu, E. Che, L. Hu, Q. Zhang and T. Jiang, et al., Nanomedicine, 2015, 11, 313-327.

31 S. F. Kiew, L. V. Kiew, H. B. Lee, T. Imae and L. Y. Chung, J. Controlled Release, 2016, 226, 217-228.

32 S. Parveen, R. Misra and S. K. Sahoo, Nanomedicine, 2012, 8, 147-166.

33 F. Masood, Mater. Sci. Eng., C, 2016, 60, 569-578.

$34 \mathrm{~J} . \mathrm{Li}$ and D. J. Mooney, Nat. Rev. Mater., 2016, 1, 16071.

35 R. Dimatteo, N. J. Darling and T. Segura, Adv. Drug Delivery Rev., 2018, 127, 167-184.

36 A. Fakhari and J. Anand Subramony, J. Controlled Release, 2015, 220, 465-475.

37 B. Shen, D. Lu, W. Zhai and W. Zheng, J. Mater. Chem. C, 2013, 1, 50-53.

38 A. Deb and R. Vimala, J. Drug Delivery Sci. Technol., 2018, 43, 333-342.

39 A. Deb, N. G. Andrews and V. Raghavan, Int. J. Biol. Macromol., 2018, 113, 515-525.

40 T. H. Tran, H. T. Nguyen, T. T. Pham, J. Y. Choi, H.-G. Choi, C. S. Yong and J. O. Kim, ACS Appl. Mater. Interfaces, 2015, 7, 28647-28655.

41 Y.-S. Huang, Y.-J. Lu and J.-P. Chen, J. Magn. Magn. Mater., 2017, 427, 34-40.

42 M. Hashemi, A. Yadegari, G. Yazdanpanah, S. Jabbehdari, M. Omidi and L. Tayebi, RSC Adv., 2016, 6, 74072-74084.

43 Z. Xu, S. Zhu, M. Wang, Y. Li, P. Shi and X. Huang, ACS Appl. Mater. Interfaces, 2015, 7, 1355-1363. 
44 F. Nasrollahi, J. Varshosaz, A. A. Khodadadi, S. Lim and A. Jahanian-Najafabadi, ACS Appl. Mater. Interfaces, 2016, 8, 13282-13293.

45 Y. Lv, L. Tao, S. W. Annie Bligh, H. Yang, Q. Pan and L. Zhu, Mater. Sci. Eng., C, 2016, 59, 652-660.

46 L. He, S. Sarkar, A. Barras, R. Boukherroub, S. Szunerits and D. Mandler, Chem. Commun., 2017, 53, 4022-4025.

47 D. de Melo-Diogo, E. C. Costa, C. G. Alves, R. Lima-Sousa, P. Ferreira, R. O. Louro and I. J. Correia, Eur. J. Pharm. Biopharm., 2018, 131, 162-169.

48 Y. T. Fong, C.-H. Chen and J.-P. Chen, Nanomaterials, 2017, 7, E388.

49 W. Zhang, Z. Guo, D. Huang, Z. Liu, X. Guo and H. Zhong, Biomaterials, 2011, 32, 8555-8561.

50 K. Yang, L. Feng and Z. Liu, Adv. Drug Delivery Rev., 2016, 105, 228-241.

51 Q. Zhang, Z. Wu, N. Li, Y. Pu, B. Wang, T. Zhang and J. Tao, Mater. Sci. Eng., C, 2017, 77, 1363-1375.

52 Z. Hasanzade and H. Raissi, Appl. Surf. Sci., 2017, 422, 1030-1041.

53 M. Mahdavi, F. Rahmani and S. Nouranian, J. Mater. Chem. B, 2016, 4, 7441-7451.

54 F. Safdari, H. Raissi, M. Shahabi and M. Zaboli, J. Inorg. Organomet. Polym., 2017, 27, 805-817.

55 Z. Hasanzade and H. Raissi, J. Mol. Model., 2017, 23, 36.

56 M. W. Tibbitt, J. E. Dahlman and R. Langer, J. Am. Chem. Soc., 2016, 138, 704-717.

57 A. P. Mathew, S. Uthaman, K.-H. Cho, C.-S. Cho and I.-K. Park, Int. J. Biol. Macromol., 2018, 110, 17-29.

58 K. Park, J. Controlled Release, 2016, 240, 2-8.

59 J. Huang, W.-J. Wang, B.-G. Li and S. Zhu, Macromol. Mater. Eng., 2013, 298, 391-399.

60 H. Hezaveh and I. I. Muhamad, J. Taiwan Inst. Chem. Eng., 2013, 44, 182-191.

61 A. F. Dexter, N. L. Fletcher, R. G. Creasey, F. Filardo, M. W. Boehm and K. S. Jack, RSC Adv., 2017, 7, 27260-27271.

62 K. Rajagopal, B. Ozbas, D. J. Pochan and J. P. Schneider, Eur. Biophys. J., 2006, 35, 162-169.

63 M. C. Branco, D. J. Pochan, N. J. Wagner and J. P. Schneider, Biomaterials, 2009, 30, 1339-1347.

64 L. Haines-Butterick, K. Rajagopal, M. Branco, D. Salick, R. Rughani, M. Pilarz, M. S. Lamm, D. J. Pochan and J. P. Schneider, Proc. Natl. Acad. Sci. U. S. A., 2007, 104, 7791-7796.

65 Y. Chen, H. X. Gan and Y. W. Tong, Macromolecules, 2015, 48, 2647-2653.

66 P. Kumar, V. Pillay, G. Modi, Y. E. Choonara, L. C. du Toit and D. Naidoo, Recent Pat. Drug Delivery Formulation, 2011, 5, 24-51.

67 A. Vashist, A. Kaushik, A. Ghosal, J. Bala, R. NikkhahMoshaie, W. A. Wani, P. Manickam and M. Nair, Gels, 2018, 4, 75.

68 M. Liu, P. Huang, W. Wang, Z. Feng, J. Zhang, L. Deng and A. Dong, J. Mater. Chem. B, 2019, 7, 2667-2677.

69 J. Basso, A. Miranda, S. Nunes, T. Cova, J. Sousa, C. Vitorino and A. Pais, Gels, 2018, 4, 62.
70 M. Constantin, S.-M. Bucatariu, F. Doroftei and G. Fundueanu, Carbohydr. Polym., 2017, 157, 493-502.

71 D. Liu, Y. Chen, X. Feng, M. Deng, G. Xie, J. Wang, L. Zhang, Q. Liu and P. Yuan, Colloids Surf., B, 2014, 113, 158-168.

72 C. Wang, G. Zhang, G. Liu, J. Hu and S. Liu, J. Controlled Release, 2017, 259, 149-159.

73 T. Lammers, V. Subr, K. Ulbrich, P. Peschke, P. E. Huber, W. E. Hennink and G. Storm, Biomaterials, 2009, 30, 3466-3475.

74 S. R. Leonard, A. R. Cormier, X. Pang, M. I. Zimmerman, H.-X. Zhou and A. K. Paravastu, Biophys. J., 2013, 105, 222-230.

75 C. Yan, A. Altunbas, T. Yucel, R. P. Nagarkar, J. P. Schneider and D. J. Pochan, Soft Matter, 2010, 6, 5143-5156.

76 N. He, O. Yildiz, Q. Pan, J. Zhu, X. Zhang, P. D. Bradford and W. Gao, J. Power Sources, 2017, 343, 492-501.

77 E. Kaiser, R. L. Colescott, C. D. Bossinger and P. I. Cook, Anal. Biochem., 1970, 34, 595-598.

78 A. M. Baptista, V. H. Teixeira and C. M. Soares, J. Chem. Phys., 2002, 117, 4184-4200.

79 A. Adnan, R. Lam, H. Chen, J. Lee, D. J. Schaffer, A. S. Barnard, G. C. Schatz, D. Ho and W. K. Liu, Mol. Pharmaceutics, 2011, 8, 368-374.

80 L. J. Henderson, Am. J. Physiol., 1908, 21, 173-179.

81 K. A. Hasselbalch, Die Berechnung der Wasserstoffzahl des Blutes aus der freien und gebundenen Kohlensäure desselben, und die Sauerstoffbindung des Blutes als Funktion der Wasserstoffzahl, Julius Springer, Berlin, 1916.

82 B. Konkena and S. Vasudevan, J. Phys. Chem. Lett., 2012, 3, 867-872.

83 G. Speelmans, R. W. H. M. Staffhorst, B. de Kruijff and F. A. de Wolf, Biochemistry, 1994, 33, 13761-13768.

84 C.-J. Shih, S. Lin, R. Sharma, M. S. Strano and D. Blankschtein, Langmuir, 2011, 28, 235.

85 A. Lerf, H. He, M. Forster and J. Klinowski, J. Phys. Chem. B, 1998, 102, 4477-4482.

86 J. Wang, R. M. Wolf, J. W. Caldwell, P. A. Kollman and D. A. Case, J. Comput. Chem., 2004, 25, 1157-1174.

87 C. I. Bayly, P. Cieplak, W. Cornell and P. A. Kollman, J. Phys. Chem., 1993, 97, 10269-10280.

88 F.-Y. Dupradeau, A. Pigache, T. Zaffran, C. Savineau, R. Lelong, N. Grivel, D. Lelong, W. Rosanski and P. Cieplak, Phys. Chem. Chem. Phys., 2010, 12, 7821.

89 E. Vanquelef, S. Simon, G. Marquant, E. Garcia, G. Klimerak, J. C. Delepine, P. Cieplak and F.-Y. Dupradeau, Nucleic Acids Res., 2011, 39, W511-W517.

90 A. Jakalian, B. L. Bush, D. B. Jack and C. I. Bayly, J. Comput. Chem., 2000, 21, 132-146.

91 A. Jakalian, D. B. Jack and C. I. Bayly, J. Comput. Chem., 2002, 23, 1623-1641.

92 D. Stauffer, N. Dragneva, W. B. Floriano, R. C. Mawhinney, G. Fanchini, S. French and O. Rubel, J. Chem. Phys., 2014, 141, 044705.

93 H. Lei, X. Wang and C. Wu, J. Mol. Graphics Modell., 2012, 38, 279-289. 
94 J. Wang, W. Wang, P. A. Kollman and D. A. Case, Abstr. Pap., Am. Chem. Soc., 2001, 222, U403.

95 L. Martínez, R. Andrade, E. G. Birgin and J. M. Martínez, J. Comput. Chem., 2009, 30, 2157-2164.

96 W. L. Jorgensen, J. Chandrasekhar, J. D. Madura, R. W. Impey and M. L. Klein, J. Chem. Phys., 1983, 79, 926.

97 J. E. Basconi and M. R. Shirts, J. Chem. Theory Comput., 2013, 9, 2887-2899.

98 K. R. Karnati and Y. Wang, Phys. Chem. Chem. Phys., 2018, 20, 9389-9400.

99 A. Yang, J. Li, C. Zhang, W. Zhang and N. Ma, Appl. Surf. Sci., 2015, 346, 443-450.

100 T. Szabó, O. Berkesi and I. Dékány, Carbon, 2005, 43, 3186-3189.

101 S. Chen, Y. Itoh, T. Masuda, S. Shimizu, J. Zhao, J. Ma, S. Nakamura, K. Okuro, H. Noguchi and K. Uosaki, et al., Science, 2015, 348, 555-559.
102 Z. Yue, C. Li, G. A. Voth and J. M. J. Swanson, J. Am. Chem. Soc., 2019, 141, 13421-13433.

103 J. Hong, R. J. Hamers, J. A. Pedersen and Q. Cui, J. Phys. Chem. C, 2017, 121, 3584-3596.

104 M. Vatanparast and Z. Shariatinia, J. Mol. Graphics Modell., 2019, 89, 50-59.

105 Y. Hu, T. Guo, X. Ye, Q. Li, M. Guo, H. Liu and Z. Wu, Chem. Eng. J., 2013, 228, 392-397.

106 M. Li, C. Liu, Y. Xie, H. Cao, H. Zhao and Y. Zhang, Carbon, 2014, 66, 302-311.

107 J. D. Schneible, A. Singhal, R. L. Lilova, C. K. Hall, A. Grafmüller and S. Menegatti, Biomacromolecules, 2019, 20, 3126-3141.

108 T.-C. Chou, Am. J. Cancer Res., 2011, 1, 925-954.

109 T.-C. Chou, Cancer Res., 2010, 70, 440-446.

110 D. R. Budman, A. Calabro, L. Rosen and M. Lesser, Anticancer Drugs, 2012, 23, 272-279. 